\title{
The influence of dynamic soil-structure interaction on traffic induced vibrations in buildings
}

\author{
S. François*, L. Pyl, H.R. Masoumi, G. Degrande \\ KU Leuven, Department of Civil Engineering, Kasteelpark Arenberg 40, B-3001 Leuven, Belgium
}

\begin{abstract}
This paper deals with the dynamic response of buildings due to traffic induced wave fields. The response of a two-storey single family dwelling due to the passage of a two-axle truck on a traffic plateau is computed with a model that fully accounts for the dynamic interaction between the soil and the structure. The results of three cases where the structure is founded on a slab foundation, a strip foundation and a box foundation are calculated and a comprehensive analysis of the dynamic structural response is performed. A methodology is also proposed to calculate the structural response, neglecting the effects of dynamic soil-structure interaction. A comparison with the results of calculations where dynamic soil-structure interaction is accounted for shows that a good approximation is obtained in the case of a rigid structure resting on a soft soil.
\end{abstract}

Keywords: Dynamic soil-structure interaction; traffic induced vibrations in buildings.

\section{Introduction}

Traffic induced vibrations in buildings are caused by moving vehicles on an uneven road or track and have become an important environmental issue. Vibrations emitted from roads or railway tracks are mainly dominated by Rayleigh waves that impinge on the foundation of nearby buildings, resulting in structural vibrations that may cause malfunctioning of sensitive equipment, discomfort to people and structural damage.

The development of three-dimensional numerical models for the prediction of traffic induced incident wave fields and the structural response has recently received considerable attention. The soil-structure interaction problem at both the source and receiver side is solved using general techniques for the dynamic analysis of coupled soil-structure systems discussed by Beskos et al. [4], Veletsos and Meek [34], Antes and Spyrakos [2], Luco [20], Wolf et al. [36, 37] and Aubry et al. [3], among others.

Numerical models for both road $[13,15,16]$ and railway traffic $[12,29-32]$ induced vibrations have been formulated, and the more general problem of moving loads on an elastic half- space has been considered $[1,22,23,33]$. In most of these models, use is made of the longitudinal invariance of the road or track which limits the computational effort [9].

In the field of earthquake engineering, structures of high societal or economical importance such as nuclear power plants, arch dams, hospitals and (long span) bridges demand for detailed design accounting for dynamic soil- structure interaction. However, the incorporation of dynamic soil-structure interaction in the analysis is quite demanding from the computational point of view. Therefore, it is important to investigate the relative importance of dynamic soil-structure interaction on the response of single family dwellings excited by traffic induced vibrations. Pyl et al. [25, 27, 28] studied the response of a single family dwelling, using a coupled finite element - boundary element formulation. This is one of the first three-dimensional analyses of traffic induced vibrations in buildings that accounts for dynamic soil-structure

\footnotetext{
*Stijn François; email: stijn.francois@bwk.kuleuven.be
} 
interaction, an effect that is disregarded in most numerical models presented in the literature [24, 35]. The first objective of the paper is to define appropriate simplified methods of analysis that do not account for dynamic soil-structure interaction effects, but properly define the foundation motion in terms of the incident wave field, accounting for the relative stiffness of the structure and the soil. The dynamic soil-structure interaction effect is important for a stiff structure resting on a soft soil. For a flexible structure on a stiff soil, however, the displacements along the soil-structure interface are almost unaffected by the presence of the building. From a practical engineering point of view, it is relevant to define under which conditions these simplified methodologies can be used as an alternative to a complete dynamic soil-structure interaction analysis.

Therefore, a two storey single family dwelling is considered, embedded in a homogeneous halfspace, and excited by a wave field caused by the passage of a two axle truck on a traffic plateau on the road in front of the building. As the type of foundation and its embedment ratio are expected to have an important influence on the impedance of the foundation, both in terms of stiffness and damping [21], as well as on the structural response, three common foundation types are considered: a slab foundation, a strip foundation and a box foundation. The structural response for these three cases is evaluated by means of the model proposed by Pyl et al. [25, 27, 28] that accounts for dynamic soil-structure interaction, as well as under the assumption that the latter effect can be disregarded, in which case the base motion of the structure must be properly defined.

\section{Numerical prediction model}

In this section, the numerical predicition model proposed by Pyl et al. [25, 27, 28] is briefly recapitulated. The dynamic response of a building due to the passage of a vehicle on an uneven road (figure 1), including three-dimensional dynamic soil-structure interaction, is considered. It is assumed that the dynamic road-soil interaction problem can be decoupled from the dynamic soil-structure interaction problem, resulting in two subproblems. This assumption is valid if the distance between the source and the receiver is much larger than the dominant wavelength in the soil.

\subsection{The source model}

The first subproblem is the calculation of the free field response in the soil domain $\Omega_{s}$ caused by the passage of a vehicle on the surface of an uneven road (figure 2). The road $\Omega_{r}$ is supported by the linear elastic layered halfspace $\Omega_{s}$ along the road- soil interface $\Sigma_{r s}$. The time history of the vertical dynamic axle load $g_{k}(t)$ of the $k$-th axle is derived from a two-dimensional vehicle model where it is assumed that the vehicle-road interaction is decoupled from the road-soil interaction problem. The axle loads move with a velocity $v$ along the line $\left\{x=x_{s}, y, z=z_{s}\right\}^{\mathrm{T}}$ in the positive $y$-direction.

The road-soil interaction problem is solved using the source model presented by Lombaert [13, 16-19]. The invariance of the road section with respect to the longitudinal coordinate $y$ allows for a Fourier transform to the horizontal wavenumber $k_{y}$. It is assumed that the vertical soil displacements $\tilde{u}_{s z}\left(x, k_{y}, \omega\right)$ at the roadsoil interface $\Sigma_{r s}$ can be written as follows in terms of the vertical translation $\tilde{u}_{c z}\left(k_{y}, \omega\right)$ of the centre of the rigid cross section and the rotation $\tilde{\beta}_{c y}\left(k_{y}, \omega\right)$ about this centre [19]:

$$
\tilde{u}_{s z}\left(x, k_{y}, \omega\right)=\tilde{u}_{c z}\left(k_{y}, \omega\right)+x \tilde{\beta}_{c y}\left(k_{y}, \omega\right)=\boldsymbol{\Phi}_{r}(x) \tilde{\mathbf{u}}_{r}\left(k_{y}, \omega\right)
$$

The vector $\boldsymbol{\Phi}_{r}(x)=\{1, x\}^{\mathrm{T}}$ collects the displacement modes of the interface $\Sigma_{r s}$, whereas the vector $\tilde{\mathbf{u}}_{r}\left(k_{y}, \omega\right)$ contains the unknown modal coordinates $\tilde{u}_{c z}\left(k_{y}, \omega\right)$ and $\tilde{\beta}_{c y}\left(k_{y}, \omega\right)$. These two displacement modes are referred to as the bending and torsional mode of the road-soil interface $\Sigma_{r s}$. A tilde above a variable denotes its representation in the frequency-wavenumber domain.

The equation of motion of the general dynamic interaction problem between the road and the soil is written in the frequency-wavenumber domain:

$$
\left[\tilde{\mathbf{K}}_{r}\left(k_{y}, \omega\right)+\tilde{\mathbf{K}}_{s}\left(k_{y}, \omega\right)\right] \tilde{\mathbf{u}}_{r}\left(k_{y}, \omega\right)=\tilde{\mathbf{f}}_{r}\left(k_{y}, \omega\right)
$$




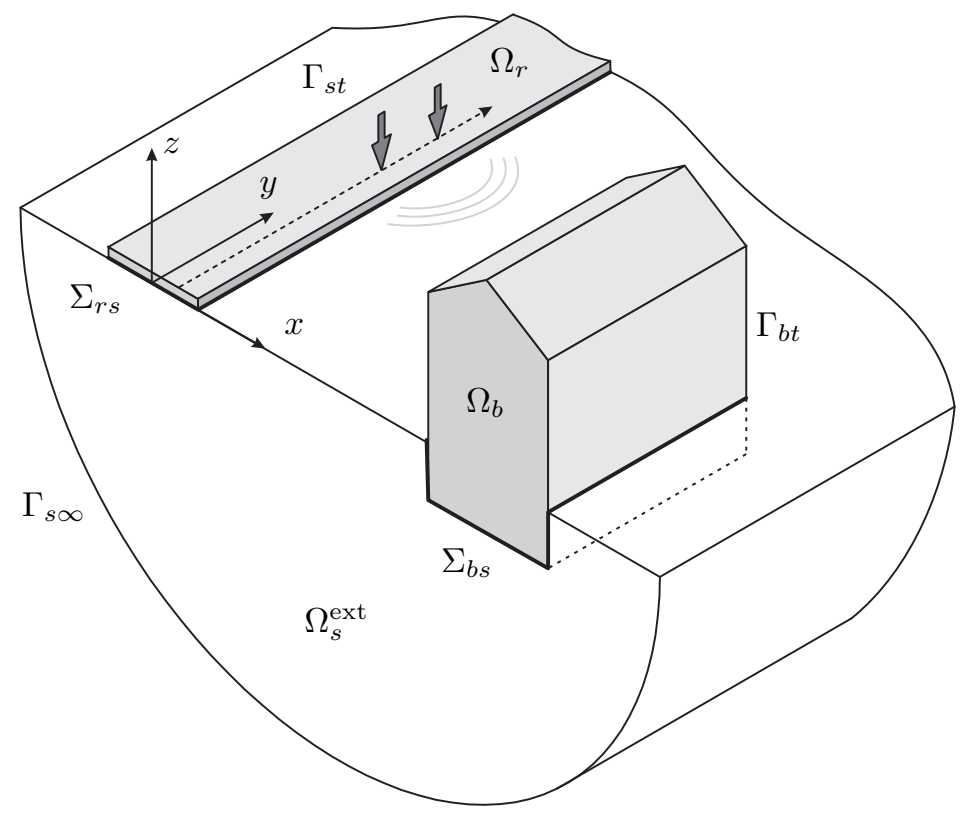

Figure 1: The dynamic road-soil-structure interaction problem.

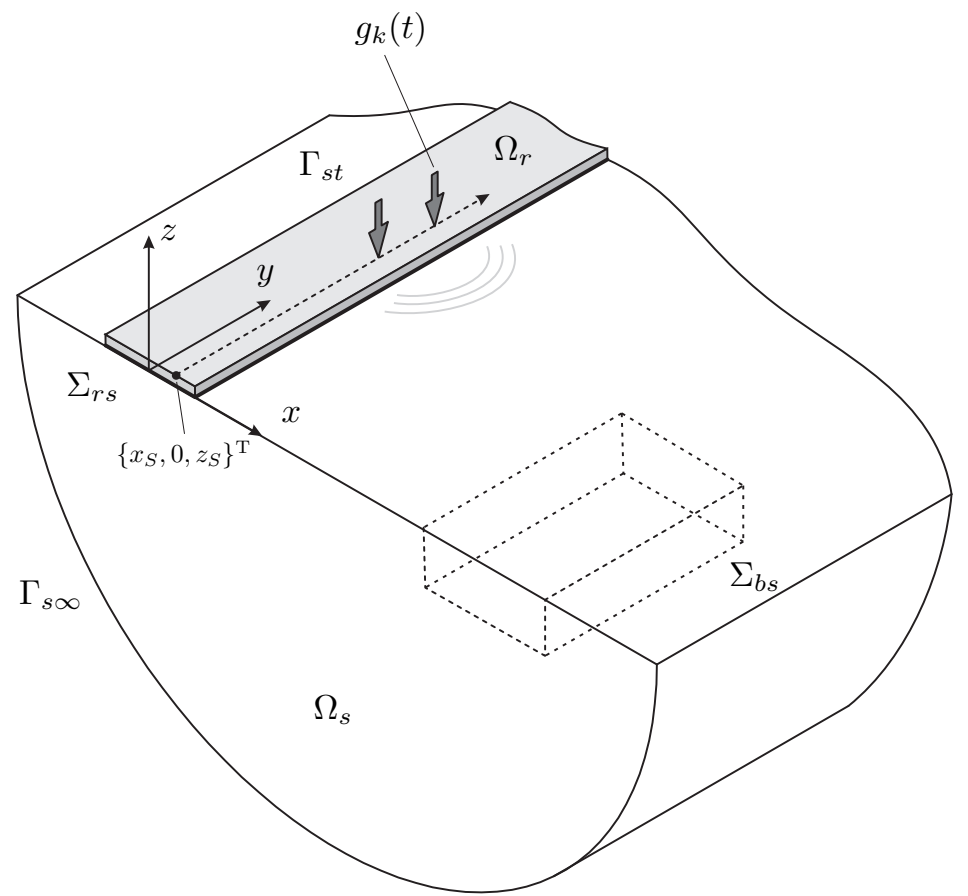

Figure 2: The dynamic road-soil interaction problem. 
$\tilde{\mathbf{K}}_{r}\left(k_{y}, \omega\right)=\operatorname{diag}\left\{E I_{x} k_{y}^{4}-\omega^{2} \rho A, G C k_{y}^{2}-\omega^{2} \rho I_{p}\right\}$ is the dynamic stiffness matrix of the road with respect to the degrees of freedom $\tilde{\mathbf{u}}_{r}\left(k_{y}, \omega\right)$, where $A$ is the road's cross section, $I_{x}$ the moment of inertia with respect to $x, C$ the torsional moment of inertia and $I_{p}$ the polar moment of inertia. $E$ is the Young's modulus, $G$ the shear modulus and $\rho$ the density of the road.

The elements of the soil impedance matrix $\tilde{\mathbf{K}}_{s}\left(k_{y}, \omega\right)$ are equal to:

$$
\tilde{K}_{s i j}\left(k_{y}, \omega\right)=\int_{\Sigma_{r s}} \Phi_{r i} \tilde{t}_{s z}\left(\Phi_{s j}\right) d \Gamma
$$

where $\Phi_{r i}$ is a vertical displacement mode of the road and $\tilde{t}_{s z}\left(\Phi_{s j}\right)$ is the vertical component of the soil traction vector $\tilde{\mathbf{t}}_{s}=\tilde{\sigma}_{s} \mathbf{n}$ on a boundary with a unit outward normal $\mathbf{n}$ due to the scattered wave field $\Phi_{s j}$ in the soil. The soil tractions are calculated by means of a boundary element method in the frequencywavenumber domain [17].

The vector $\tilde{\mathbf{f}}_{r}\left(k_{y}, \omega\right)$ represents the load vector on the road in the frequency-wavenumber domain. For a concentrated vertical impulsive load in the point $\left\{x_{S}, 0, z_{S}\right\}^{\mathrm{T}}$ (figure 2), the load vector is equal to $\left\{1, x_{S}\right\}^{\mathrm{T}}$.

The complex modal displacements $\tilde{\mathbf{u}}_{r}\left(k_{y}, \omega\right)$ result from the solution of the system of equations (2). The soil tractions $\tilde{t}_{s z}\left(\tilde{\mathbf{u}}_{s}\right)$ at the road-soil interface are calculated from these modal coordinates as $\tilde{t}_{s z}\left(\boldsymbol{\Phi}_{r}\right) \tilde{\mathbf{u}}_{r}\left(k_{y}, \omega\right)$.

The road-soil transfer function $h_{z i}(x, y, z, t)$ is defined as the displacement in the direction $\mathbf{e}_{i}$ at a point $\{x, y, z\}^{\mathrm{T}}$ of the road or the soil due to a vertical impulsive load on the road at $\left\{x_{S}, 0, z_{S}\right\}^{\mathrm{T}}$. The reciprocity theorem is used for the calculation of the road-soil transfer function $\tilde{h}_{z i}\left(x, k_{y}, z, \omega\right)$ from the soil tractions at the interface. In the load case considered, only the vertical tractions $\tilde{t}_{s z}\left(x^{\prime}, k_{y}, z^{\prime}=0, \omega\right)$ have a non-zero resultant. When the loaded area is small compared to the wavelength in the soil, it can be assumed that the horizontal tractions have a small influence on the free field displacements, so that:

$$
\tilde{h}_{z i}\left(x, k_{y}, z, \omega\right)=\int_{-B}^{B} \tilde{u}_{z i}^{G}\left(x-x^{\prime}, k_{y}, z, \omega\right) \tilde{t}_{s z}\left(x^{\prime}, k_{y}, z^{\prime}=0, \omega\right) d x^{\prime}
$$

and only the Green's function $\tilde{u}_{z i}^{G}\left(x, k_{y}, z, \omega\right)$, representing the fundamental solution for the displacement in the direction $\mathbf{e}_{i}$ due to a vertical impulsive load, is needed for the calculation of the transfer function.

Finally, the dynamic Betti-Rayleigh reciprocal theorem is used to calculate the response of the soil or the road. The invariance of the problem geometry with respect to $y$ allows to calculate the displacements as the following convolution integral of the vertical axle loads $g_{k}(t)$ and the transfer function $h_{z i}(x, y, z, t)$ between the source and the receiver:

$$
u_{s i}(x, y, z, t)=\sum_{k=1}^{n} \int_{-\infty}^{t} h_{z i}\left(x, y-y_{k}-v \tau, z, t-\tau\right) g_{k}(\tau) d \tau
$$

The representation of this solution in the frequency-wavenumber domain is:

$$
\tilde{u}_{s i}\left(x, k_{y}, z, \omega\right)=\tilde{h}_{z i}\left(x, k_{y}, z, \omega\right) \sum_{k=1}^{n} \hat{g}_{k}\left(\omega-k_{y} v\right) \exp \left(i k_{y} y_{k}\right)
$$

Note that a frequency shift $k_{y} v$ is applied to the argument of the interaction force $\hat{g}_{k}\left(\omega-k_{y} v\right)$, where $\omega$ is the frequency at the receiver, whereas $\omega-k_{y} v$ corresponds to the frequency emitted at the source. The latter will be denoted as $\tilde{\omega}$. The displacements $\hat{u}_{s i}(x, y, z, \omega)$ in the frequency domain are found as the inverse Fourier transform of equation (6):

$$
\hat{u}_{s i}(x, y, z, \omega)=\frac{1}{2 \pi} \int_{-\infty}^{+\infty} \tilde{h}_{z i}\left(x, k_{y}, z, \omega\right) \sum_{k=1}^{n} \hat{g}_{k}\left(\omega-k_{y} v\right) \exp \left[-i k_{y}\left(y-y_{k}\right)\right] d k_{y}
$$

A change of variables according to $k_{y}=(\omega-\tilde{\omega}) / v$ moves the frequency shift from the axle load to the transfer function:

$$
\hat{u}_{s i}(x, y, z, \omega)=\frac{1}{2 \pi v} \int_{-\infty}^{+\infty} \tilde{h}_{z i}\left(x, \frac{\omega-\tilde{\omega}}{v}, z, \omega\right) \sum_{k=1}^{n} \hat{g}_{k}(\tilde{\omega}) \exp \left[-i\left(\frac{\omega-\tilde{\omega}}{v}\right)\left(y-y_{k}\right)\right] d \tilde{\omega}
$$


and illustrates that traffic induced vibrations are caused by dynamic vehicle loads and wave propagation in the soil. The solution in the time domain is finally found as the inverse Fourier transformation from the circular frequency $\omega$ to the time $t$.

\subsection{The receiver model}

The second subproblem is the calculation of the response of the structure $\Omega_{b}$ due to the traffic induced incident wave field (figure 3). A subdomain formulation [3, 6-8] is employed in order to account for dynamic soil-structure interaction.

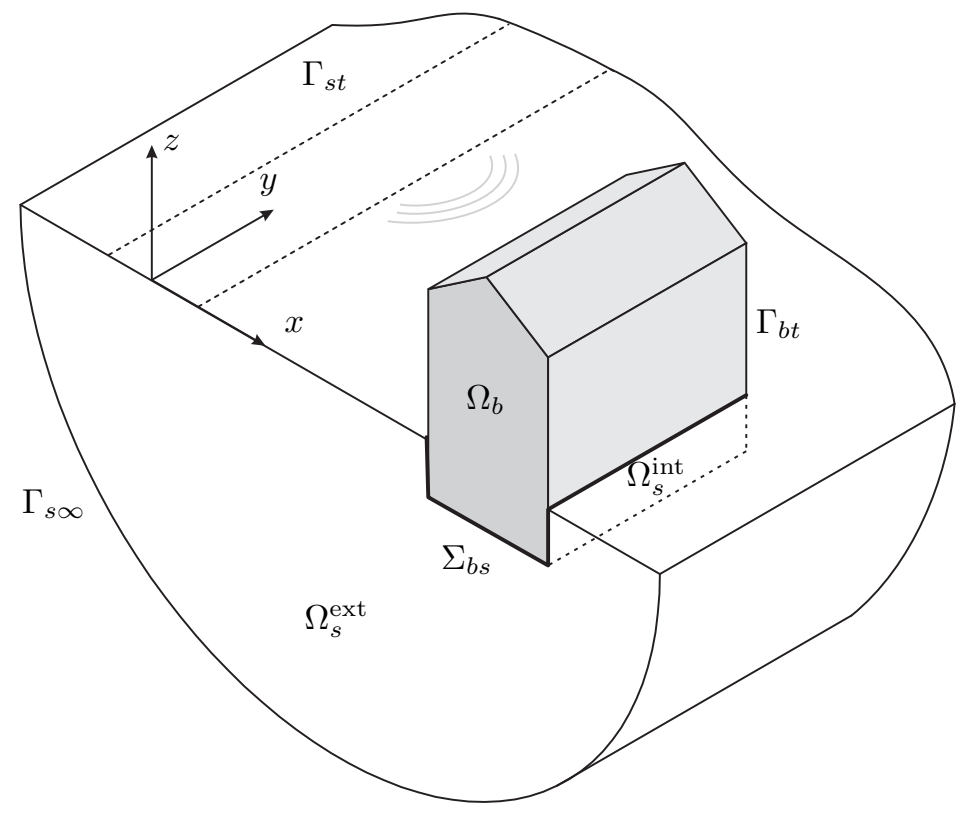

Figure 3: The dynamic soil-structure interaction problem.

The soil domain $\Omega_{s}$ is divided into the internal soil domain $\Omega_{s}^{\text {int }}=\Omega_{s} \cap \Omega_{b}$ and the external soil domain $\Omega_{s}^{\text {ext }}$. The displacement vector $\boldsymbol{u}_{s}$ in the external soil domain is decomposed into a displacement field $\boldsymbol{u}_{0}$ and a wave field $\boldsymbol{u}_{\mathrm{sc}}\left(\boldsymbol{u}_{b}\right)$ scattered by the displacements $\boldsymbol{u}_{b}$ on the interface $\Sigma_{b s}$ :

$$
\boldsymbol{u}_{s}=\boldsymbol{u}_{0}+\boldsymbol{u}_{\mathrm{sc}}\left(\boldsymbol{u}_{b}\right) \quad \text { in } \Omega_{s}^{\mathrm{ext}}
$$

The displacement field $\boldsymbol{u}_{0}$ is zero on the interface $\Sigma_{b s}$. It is further decomposed into the incident wave field $\boldsymbol{u}_{\text {inc }}$ and a locally diffracted wave field $\boldsymbol{u}_{\mathrm{d} 0}$, so that the displacement vector $\boldsymbol{u}_{s}$ becomes:

$$
\boldsymbol{u}_{s}=\boldsymbol{u}_{\mathrm{inc}}+\boldsymbol{u}_{\mathrm{d} 0}+\boldsymbol{u}_{\mathrm{sc}}\left(\boldsymbol{u}_{b}\right) \quad \text { in } \Omega_{s}^{\mathrm{ext}}
$$

The displacement field $\boldsymbol{u}_{\text {inc }}$ is defined in the soil domain $\Omega_{s}$ and evaluated by means of equation (8). The locally diffracted wave field $\boldsymbol{u}_{\mathrm{d} 0}$ is equal to $-\boldsymbol{u}_{\text {inc }}$ on the interface $\Sigma_{b s}$.

In the domain $\Omega_{b}$, the displacement vector $\boldsymbol{u}_{b}$ is discretised as $\boldsymbol{u}_{b}=\boldsymbol{N}_{b} \mathbf{u}_{\mathbf{b}}$ using globally defined finite element shape functions $\boldsymbol{N}_{b}$. The displacement vector $\mathbf{u}_{b}$ is subdivided into the displacements $\mathbf{u}_{b_{2}}$ of the foundation and the displacements $\mathbf{u}_{b_{1}}$ of the remaining part of the structure (superstructure). All degrees of freedom on the soil-structure interface $\Sigma_{b s}$ belong to the vector $\mathbf{u}_{b_{2}}$. 
The soil-structure interaction problem is solved by enforcing continuity of displacements and equilibrium of stresses on the soil-structure interface $\Sigma_{b s}$. The equilibrium equation of the coupled soil-structure system is equal to:

$$
\begin{aligned}
\left(\left[\begin{array}{ll}
\mathbf{K}_{b_{1} b_{1}} & \mathbf{K}_{b_{1} b_{2}} \\
\mathbf{K}_{b_{2} b_{1}} & \mathbf{K}_{b_{2} b_{2}}
\end{array}\right]-\omega^{2}\left[\begin{array}{ll}
\mathbf{M}_{b_{1} b_{1}} & \mathbf{M}_{b_{1} b_{2}} \\
\mathbf{M}_{b_{2} b_{1}} & \mathbf{M}_{b_{2} b_{2}}
\end{array}\right]+\left[\begin{array}{cc}
\mathbf{0} & \mathbf{0} \\
\mathbf{0} & \hat{\mathbf{K}}_{b_{2} b_{2}}^{s}(\omega)
\end{array}\right]\right) & \left\{\begin{array}{l}
\hat{\mathbf{u}}_{b_{1}}(\omega) \\
\hat{\mathbf{u}}_{b_{2}}(\omega)
\end{array}\right\} \\
& =\left\{\begin{array}{l}
\hat{\mathbf{f}}_{b_{1}}(\omega) \\
\hat{\mathbf{f}}_{b_{2}}(\omega)
\end{array}\right\}
\end{aligned}
$$

The first and second matrix on the left hand side represent the stiffness and mass matrix of the structure. These matrices are subdivided into block matrices according to the degrees of freedom $\hat{\mathbf{u}}_{b_{2}}(\omega)$ of the foundation and the degrees of freedom $\hat{\mathbf{u}}_{b_{1}}(\omega)$ of the superstructure. The dynamic stiffness matrix $\hat{\mathbf{K}}_{b_{2} b_{2}}^{s}(\omega)$ of the soil is equal to:

$$
\hat{\mathbf{K}}_{b_{2} b_{2}}^{s}(\omega)=\int_{\Sigma_{b s}} \mathbf{N}_{b_{2}}^{\mathrm{T}} \hat{\boldsymbol{t}}_{s}\left(\boldsymbol{u}_{s c}\left(\mathbf{N}_{b_{2}}\right)\right) d \Sigma
$$

where $\mathbf{N}_{b_{2}}$ are the shape functions related to the degrees of freedom $\mathbf{u}_{b_{2}}$. The vector $\hat{\mathbf{f}}_{b_{2}}(\omega)$ denotes the loading term due to the incident wave field on the interface $\Sigma_{b s}$ :

$$
\hat{\mathbf{f}}_{b_{2}}(\omega)=-\int_{\Sigma_{b s}} \mathbf{N}_{b_{2}}^{\mathrm{T}} \hat{\boldsymbol{t}}_{s}\left(\boldsymbol{u}_{\mathrm{inc}}+\boldsymbol{u}_{\mathrm{d} 0}\right) d \Sigma
$$

The following Craig-Bampton decomposition is used [10] in order to limit the computational effort:

$$
\left\{\begin{array}{c}
\hat{\mathbf{u}}_{b_{1}}(\omega) \\
\hat{\mathbf{u}}_{b_{2}}(\omega)
\end{array}\right\}=\left[\begin{array}{cc}
\boldsymbol{\Psi}_{b_{1}} & \boldsymbol{\Psi}_{b_{1}}^{s} \\
\mathbf{0} & \boldsymbol{\Psi}_{b_{2}}
\end{array}\right]\left\{\begin{array}{c}
\hat{\boldsymbol{\alpha}}_{b_{1}}(\omega) \\
\hat{\boldsymbol{\alpha}}_{b_{2}}(\omega)
\end{array}\right\}=\Psi_{b} \hat{\boldsymbol{\alpha}}_{b}(\omega)
$$

The eigenmodes $\boldsymbol{\Psi}_{b_{1}}$ of the superstructure clamped at the base are found as the solution of the following generalised eigenvalue problem:

$$
\mathbf{K}_{b_{1} b_{1}} \boldsymbol{\Psi}_{b_{1}}-\lambda \mathbf{M}_{b_{1} b_{1}} \boldsymbol{\Psi}_{b_{1}}=0
$$

whereas the free foundation modes $\boldsymbol{\Psi}_{b_{2}}$ are the solution of the generalised eigenvalue problem:

$$
\mathbf{K}_{b_{2} b_{2}}^{\prime} \Psi_{b_{2}}-\lambda \mathbf{M}_{b_{2} b_{2}}^{\prime} \Psi_{b_{2}}=0
$$

The matrices $\mathbf{K}_{b_{2} b_{2}}^{\prime}$ and $\mathbf{M}_{b_{2} b_{2}}^{\prime}$ are the stiffness and mass matrices of the foundation not connected to the structure. The quasi-static transmission $\boldsymbol{\Psi}_{b_{1}}^{s}$ of the foundation modes $\boldsymbol{\Psi}_{b_{2}}$ is equal to:

$$
\boldsymbol{\Psi}_{b_{1}}^{s}=-\mathbf{K}_{b_{1} b_{1}}^{-1} \mathbf{K}_{b_{1} b_{2}} \boldsymbol{\Psi}_{b_{2}}
$$

The corresponding modal coordinates are collected in the vectors $\hat{\boldsymbol{\alpha}}_{b_{1}}(\omega)$ and $\hat{\boldsymbol{\alpha}}_{b_{2}}(\omega)$.

Introduction of the decomposition (14) into equation (11) results in:

$$
\begin{aligned}
& \left(\left[\begin{array}{cc}
\boldsymbol{\Lambda}_{k k} & \mathbf{0} \\
\mathbf{0} & \mathbf{\Psi}_{b_{2}}^{\mathrm{T}}\left(\mathbf{K}_{b_{2} b_{2}}-\mathbf{K}_{b_{2} b_{1}} \mathbf{K}_{b_{1} b_{1}}^{-1} \mathbf{K}_{b_{1} b_{2}}\right) \mathbf{\Psi}_{b_{2}}
\end{array}\right]\right.
\end{aligned}
$$

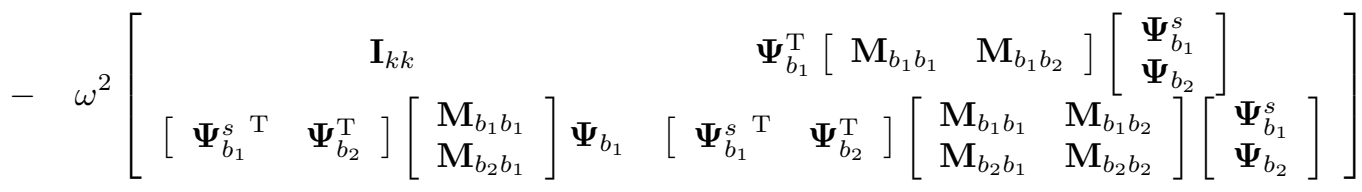

$$
\begin{aligned}
& \left.+\left[\begin{array}{cc}
\mathbf{0} & \mathbf{0} \\
\mathbf{0} & \boldsymbol{\Psi}_{b_{2}}^{\mathrm{T}} \hat{\mathbf{K}}_{b_{2} b_{2}}^{s}(\omega) \boldsymbol{\Psi}_{b_{2}}
\end{array}\right]\right)\left\{\begin{array}{c}
\hat{\boldsymbol{\alpha}}_{b_{1}}(\omega) \\
\hat{\boldsymbol{\alpha}}_{b_{2}}(\omega)
\end{array}\right\}=\left[\begin{array}{c}
\boldsymbol{\Psi}_{b_{1}}^{\mathrm{T}} \hat{\mathbf{f}}_{b_{1}}(\omega) \\
\boldsymbol{\Psi}_{b_{1}}^{s} \mathrm{~T}_{b_{1}}(\omega)+\boldsymbol{\Psi}_{b_{2}}^{\mathrm{T}} \hat{\mathbf{f}}_{b_{2}}(\omega)
\end{array}\right]
\end{aligned}
$$


where $\boldsymbol{\Lambda}_{k k}=\operatorname{diag}\left(\omega_{j}^{2}\right)$ is a $k$-dimensional diagonal matrix containing the squares of the eigenfrequencies $\omega_{j}(j=1, \ldots, k)$ of the superstructure clamped at the base. The matrix $\mathbf{I}_{k k}$ is a $k$-dimensional unit matrix reflecting the orthogonality of the modes $\mathbf{\Psi}_{b_{1}}$ with respect to the mass matrix $\mathbf{M}_{b_{1} b_{1}}$.

The projection $\boldsymbol{\Psi}_{b_{2}}^{\mathrm{T}} \hat{\mathbf{K}}_{b_{2} b_{2}}^{s}(\omega) \boldsymbol{\Psi}_{b_{2}}$ of the dynamic soil stiffness matrix on the free foundation modes is written by means of equation (12) as:

$$
\boldsymbol{\Psi}_{b_{2}}^{\mathrm{T}} \hat{\mathbf{K}}_{b_{2} b_{2}}^{s}(\omega) \boldsymbol{\Psi}_{b_{2}}=\int_{\Sigma_{b s}}\left(\mathbf{N}_{b_{2}} \boldsymbol{\Psi}_{b_{2}}\right)^{\mathrm{T}} \hat{\boldsymbol{t}}_{s}\left(\boldsymbol{u}_{s c}\left(\mathbf{N}_{b_{2}} \boldsymbol{\Psi}_{b_{2}}\right)\right) d \Sigma
$$

Similarly, the modal force $\boldsymbol{\Psi}_{b_{2}}^{\mathrm{T}} \hat{\mathbf{f}}_{b_{2}}(\omega)$ is written as:

$$
\boldsymbol{\Psi}_{b_{2}}^{\mathrm{T}} \hat{\mathbf{f}}_{b_{2}}(\omega)=-\int_{\Sigma_{b s}}\left(\boldsymbol{\Psi}_{b_{2}} \mathbf{N}_{b_{2}}\right)^{\mathrm{T}} \hat{\boldsymbol{t}}_{s}\left(\boldsymbol{u}_{\mathrm{inc}}+\boldsymbol{u}_{\mathrm{d} 0}\right) d \Sigma
$$

Equations (19) and (20) are evaluated by means of a frequency domain boundary element method [6-8]. This is computationally more efficient than the calulation of the matrices $\hat{\mathbf{K}}_{b_{2} b_{2}}^{s}(\omega)$ and $\hat{\mathbf{f}}_{b_{2}}(\omega)$ by means of equations (12) and (13) followed by a projection on the foundation modes $\boldsymbol{\Psi}_{b_{2}}$.

The present substructuring technique offers the advantage that the foundation and the superstructure can be analysed independently. In particular, the recomputation of the dynamic soil stiffness matrix $\boldsymbol{\Psi}_{b_{2}}^{\mathrm{T}} \hat{\mathbf{K}}_{b_{2} b_{2}}^{s}(\omega) \boldsymbol{\Psi}_{b_{2}}$ and the load vector $\boldsymbol{\Psi}_{b_{2}}^{\mathrm{T}} \hat{\mathbf{f}}_{b_{2}}(\omega)$ associated with the incident wave field are avoided whenever the properties of the superstructure are changed.

In the following, proportional modal damping is introduced and the term

$$
i \omega\left[\begin{array}{cc}
\mathbf{C}_{b_{1} b_{1}}^{\prime} & \mathbf{0} \\
\mathbf{0} & \mathbf{C}_{b_{2} b_{2}}^{\prime}
\end{array}\right]\left\{\begin{array}{c}
\hat{\boldsymbol{\alpha}}_{b_{1}}(\omega) \\
\hat{\boldsymbol{\alpha}}_{b_{2}}(\omega)
\end{array}\right\}
$$

is added to the left hand side of equation (18). $\mathbf{C}_{b_{1} b_{1}}^{\prime}=\operatorname{diag}\left(2 \xi_{j} \omega_{j}\right)$ is a $k$-dimensional diagonal matrix where $\xi_{j}(j=1, \ldots, k)$ are the modal damping ratios of the superstructure clamped at the base. Similarly, $\mathbf{C}_{b_{2} b_{2}}^{\prime}=\operatorname{diag}\left(2 \xi_{j} \omega_{j}\right)$ is a $l$-dimensional diagonal matrix where $\xi_{j}(j=1, \ldots, l)$ are modal damping ratios of the free foundation.

\section{The road-soil interaction problem}

The response of a two-storey single family dwelling due to the passage of a two-axle truck on a traffic plateau is considered [14]. Figure 4 shows the road, the traffic plateau and the soil-structure interface. The origin of the Cartesian frame of reference is located at the centre of the road in the middle of the traffic plateau. The road has a width $2 B=4 \mathrm{~m}$ and consists of three layers: an asphalt top layer, a layer of crushed stone and a crushed concrete subbase layer (table 1). A traffic plateau with a top length $L=10 \mathrm{~m}$, a height $H=0.12 \mathrm{~m}$ and sine-shaped ramps with a length $l=1.2 \mathrm{~m}$ is located on the road surface. The road is supported by a homogeneous halfspace with a shear wave velocity $C_{s}=200 \mathrm{~m} / \mathrm{s}$, a longitudinal wave velocity $C_{p}=400 \mathrm{~m} / \mathrm{s}$, a density $\rho=1750 \mathrm{~kg} / \mathrm{m}^{3}$, a Poisson's ratio $\nu=1 / 3$ and a material damping ratio $\beta=0.025$ in deviatoric and volumetric deformation.

Table 1: The material properties of the road.

\begin{tabular}{cccccc}
\hline Layer & Type & $\begin{array}{c}d \\
{[\mathrm{~m}]}\end{array}$ & $\begin{array}{c}E \\
{\left[\times 10^{6} \mathrm{~N} / \mathrm{m}^{2}\right]}\end{array}$ & $\begin{array}{c}\nu \\
{[-]}\end{array}$ & $\begin{array}{c}\rho \\
{\left[\mathrm{kg} / \mathrm{m}^{3}\right]}\end{array}$ \\
\hline 1 & Asphalt & 0.15 & 9150 & $1 / 3$ & 2100 \\
2 & Crushed stone & 0.20 & 500 & $1 / 2$ & 2000 \\
3 & Crushed concrete & 0.25 & 200 & $1 / 2$ & 1800 \\
\hline
\end{tabular}

A two-axle truck with a wheel base of $5.2 \mathrm{~m}$ passes at a vehicle speed $v=50 \mathrm{~km} / \mathrm{h}$ on the traffic plateau. At the time $t=0$, the front axle of the vehicle is located at $x_{S}=1 \mathrm{~m}, y=0 \mathrm{~m}$ and $z_{S}=0 \mathrm{~m}$. The axle loads are derived from a two-dimensional $4 \mathrm{DOF}$ vehicle model and are applied at $x_{S}=B / 2=1 \mathrm{~m}$ (figure 


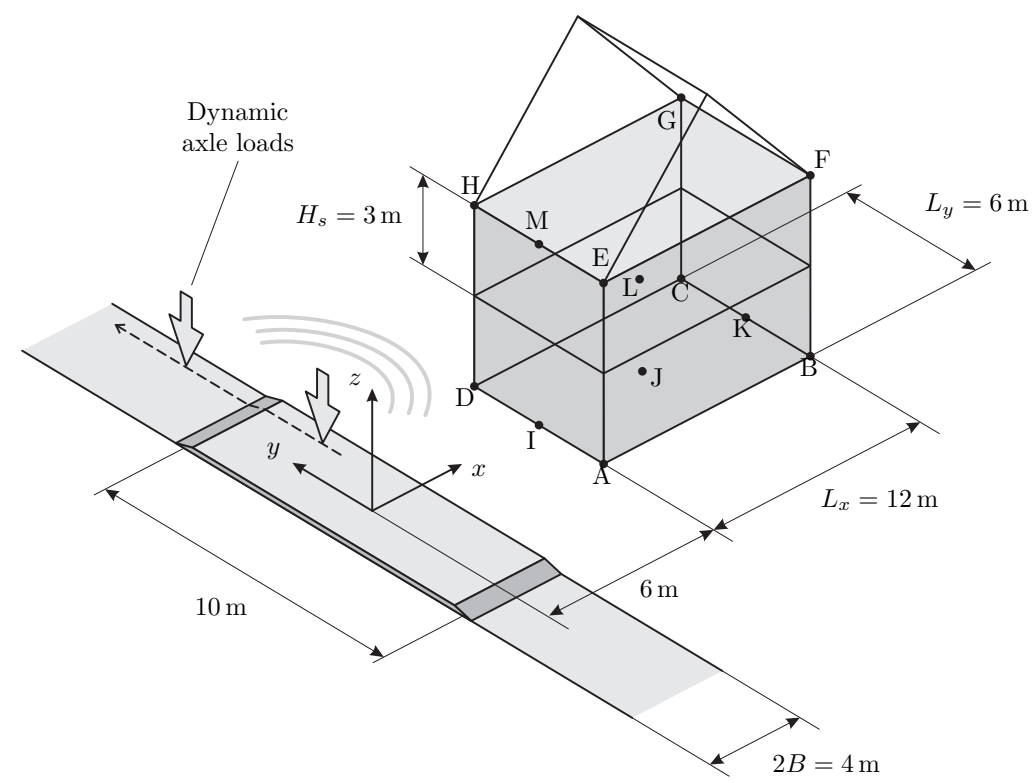

Figure 4: Geometry of the road, the traffic plateau and the structure.

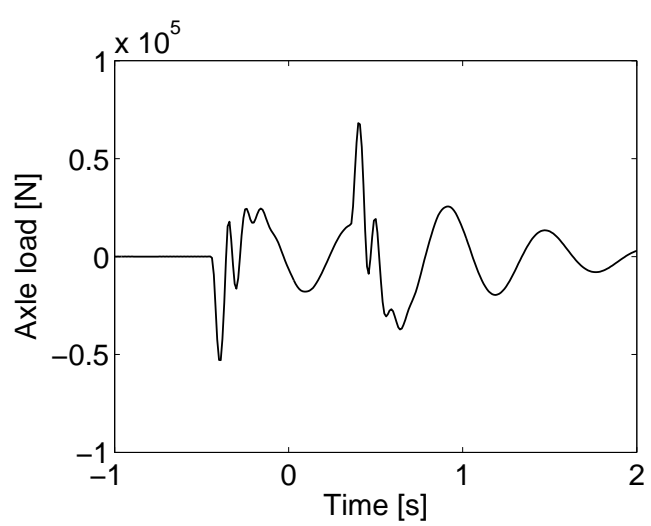

a. $g_{2}(t)$.

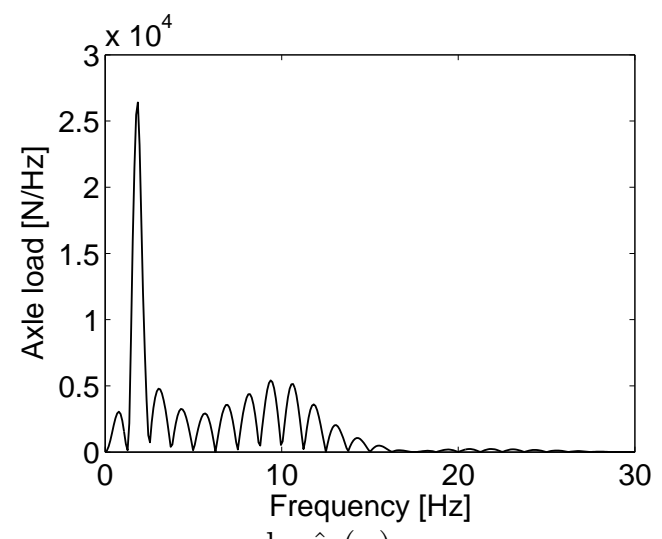

b. $\hat{g}_{2}(\omega)$.

Figure 5: (a) Time history and (b) frequency content of the front axle load due to a passage of a truck on a traffic plateau at a speed of $14 \mathrm{~m} / \mathrm{s}$.

5). The frequency content of the axle loads is dominated by the pitch and bounce mode of the front and the rear axle at $1.9 \mathrm{~Hz}$ and $1.4 \mathrm{~Hz}$ and the axle hop modes at $10.8 \mathrm{~Hz}$ and $12.4 \mathrm{~Hz}$ (figure $5 \mathrm{~b}$ ).

The model proposed by Lombaert [17-19] is used to calculate the wave field caused by the passage of the truck on the plateau. The time history of the vertical velocity in the free field (figure 6) shows four peaks corresponding to the passage of the vehicle axles on the two ramps of the traffic plateau. Whereas the pitch and bounce mode dominate the frequency content of the axle loads, the axle hop modes dominate the frequency content of the free field response.

Figure 7 shows the amplitude of the displacement vector in the free field at different time steps, corresponding to radiated wave fronts during the passage of the rear axle on the first ramp (figure 4). As the bending stiffness of the road is negligible with respect to the stiffness of the soil, cylindrical Rayleigh wave fronts can clearly be observed in the soil. 
1.
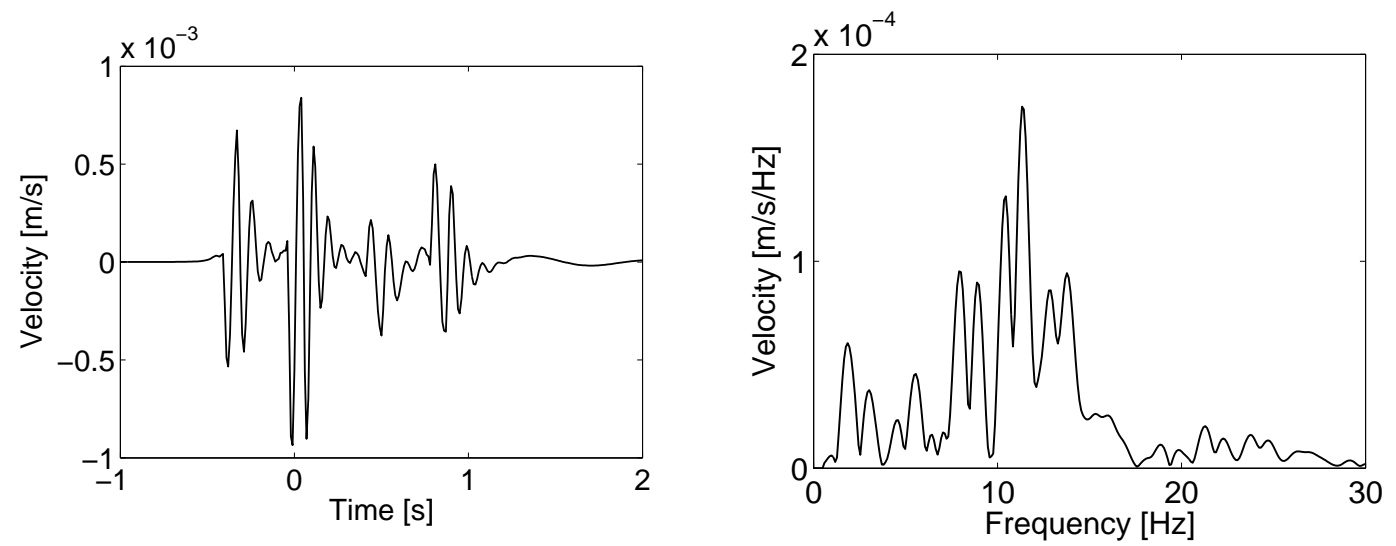

2 .
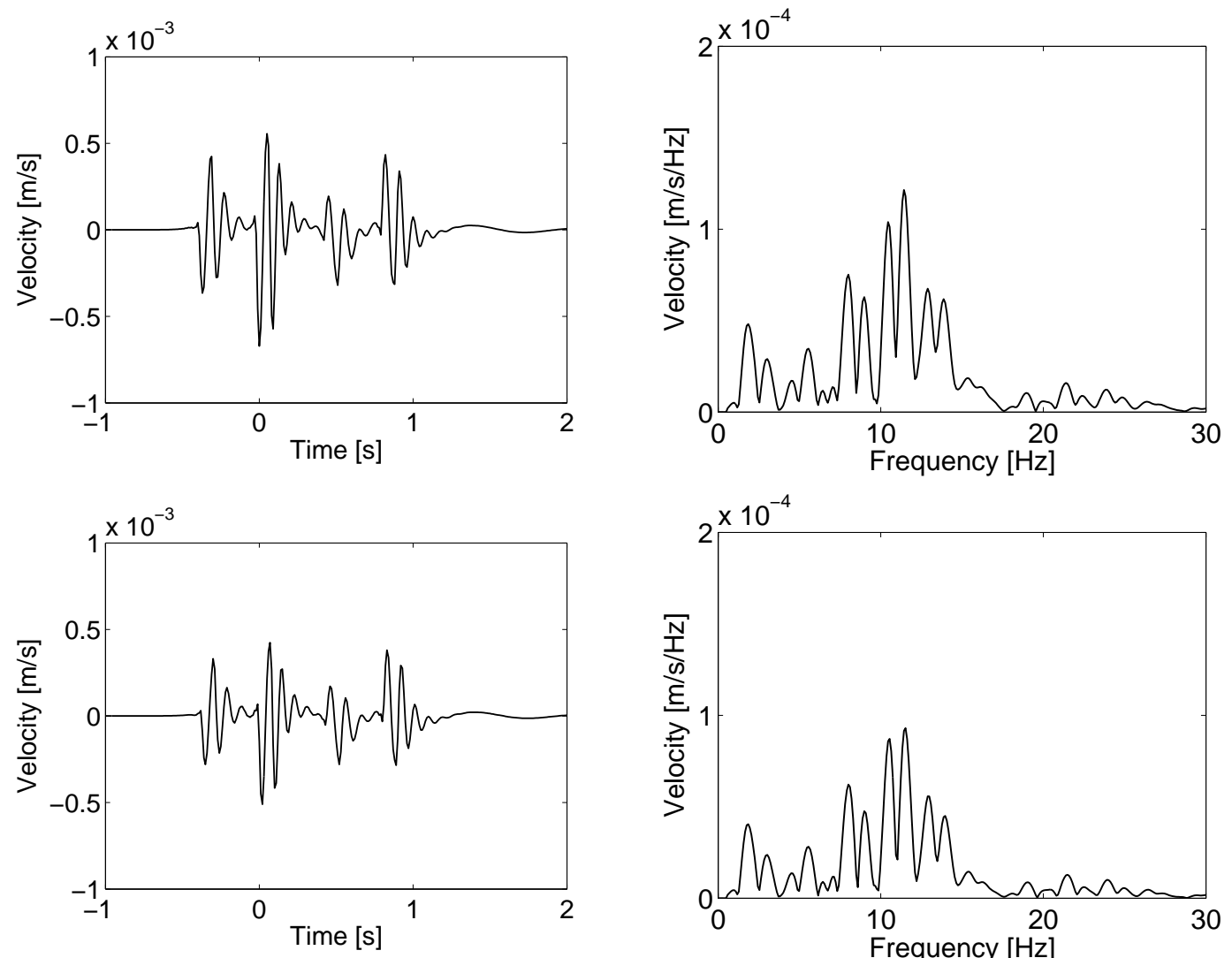

a. $v_{\text {inc } z}(t)$.

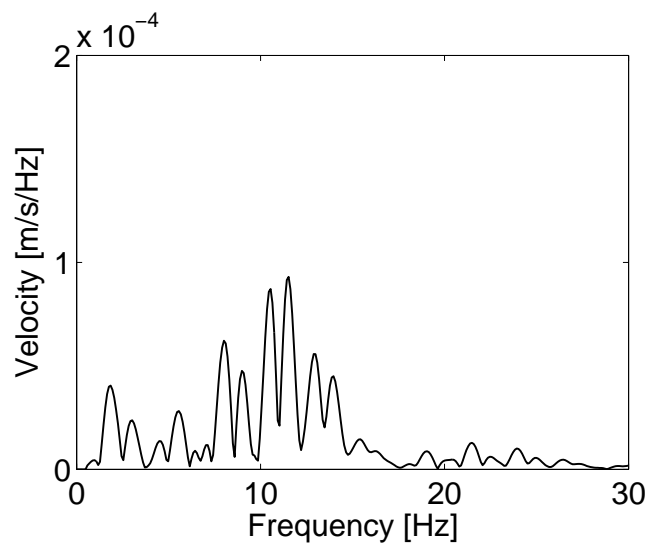

b. $\hat{v}_{\text {inc } z}(\omega)$.

Figure 6: (a) Time history and (b) frequency content of the free field vertical velocity in the points (1) I, (2) J and (3) K (figure 4) due to a passage of a truck on a traffic plateau at a speed of $14 \mathrm{~m} / \mathrm{s}$.

\section{The soil-structure interaction problem}

Figure 4 shows the two-storey structure with a storey height $H_{s}=3 \mathrm{~m}$. The structure has a length $L_{x}=12 \mathrm{~m}$ and a width $L_{y}=6 \mathrm{~m}$. The material properties of the building are summarized in table 2 . The roof structure has a total mass of $5000 \mathrm{~kg}$, which is uniformely lumped along the lines EH and FG (figure 4). Both gable ends have a mass of $1000 \mathrm{~kg}$ and are modeled as triangular mass distributions along the lines $\mathrm{EF}$ and GH (figure 4). A reduced wall thickness for walls AEHD and BFGC accounts for the presence of 


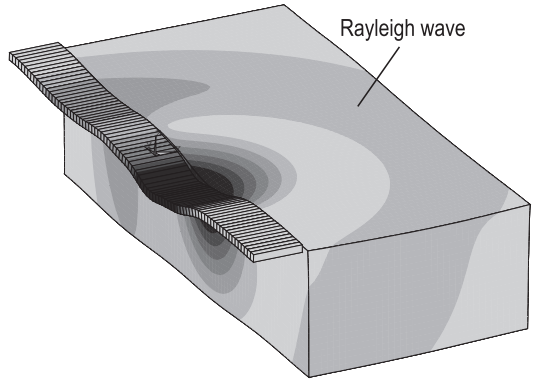

a. $t=0.08 \mathrm{~s}$.

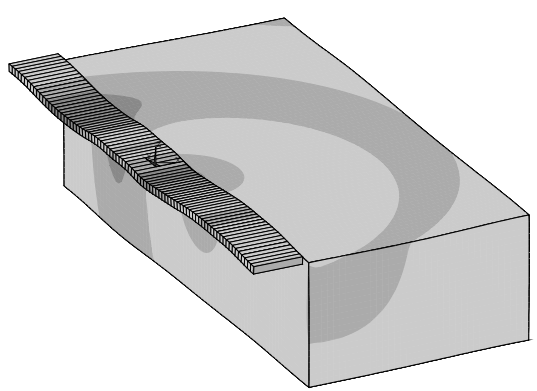

c. $t=0.16 \mathrm{~s}$.

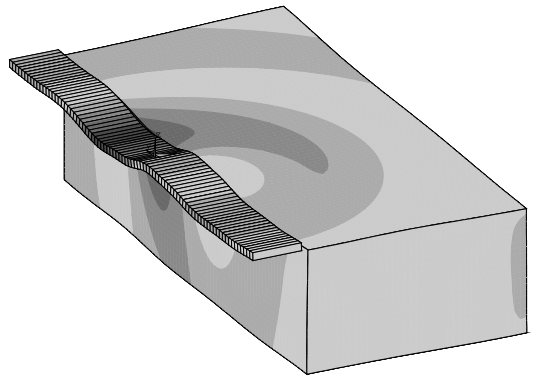

b. $t=0.12 \mathrm{~s}$.

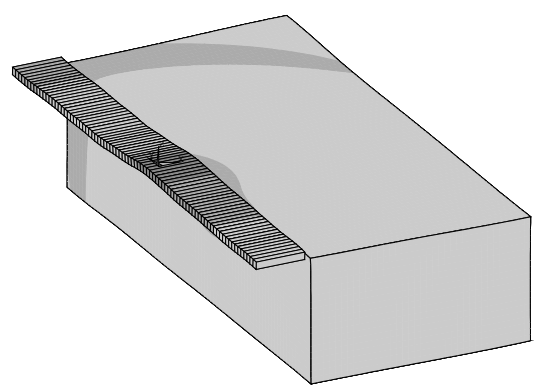

d. $t=0.20 \mathrm{~s}$.

Figure 7: Displacements in the free field at different time steps due to the passage of a truck on a traffic plateau at a speed of $14 \mathrm{~m} / \mathrm{s}$.

window and door openings.

Table 2: The material properties of the building.

\begin{tabular}{ccccc}
\hline & $d$ & $E$ & $\nu$ & $\rho$ \\
& {$[\mathrm{m}]$} & {$\left[\times 10^{6} \mathrm{~N} / \mathrm{m}^{2}\right]$} & {$[-]$} & {$\left[\mathrm{kg} / \mathrm{m}^{3}\right]$} \\
\hline Walls & 0.18 & 10500 & 0.15 & 1200 \\
Slabs & 0.20 & 33300 & 0.20 & 2500 \\
\hline
\end{tabular}

The type of foundation and its embedment ratio are expected to have an important influence on the impedance of the foundation, as well as on the structural response. Therefore, three common foundation types are considered: a slab foundation, a strip foundation and a box foundation (figure 8). All foundations consist of concrete with a Young's modulus $E=33300 \mathrm{MPa}$, a Poisson's ratio $\nu=1 / 3$ and a density $\rho=2500 \mathrm{~kg} / \mathrm{m}^{3}$. The foundation walls and slabs have a thickness of $0.3 \mathrm{~m}$. In the case of the strip and the box foundation, an additional slab ABCD is added to the superstucture.

\subsection{Finite element model}

The superstructure is modelled with 4-node quadrilateral shell elements with six degrees of freedom per node. All floors are simply supported, corresponding to hinged joints at the slab edges. The slab and the box foundation are also modelled with 4-node quadrilateral shell elements, whereas 8-node brick elements are used for the discretisation of the strip foundation.

The minimum wavelength in the slab foundation at the upper bound $f_{\max }=50 \mathrm{~Hz}$ of the frequency range of interest is equal to $\lambda_{\min }=\sqrt{2 \pi} \sqrt[4]{D / \rho d} / \sqrt{f_{\max }}=6 \mathrm{~m}$, where $D=E d^{3} / 12\left(1-\nu^{2}\right)$ is the bending stiffness 
of the slab with a thickness $d$ and a density $\rho$. The rule of thumb that recommends to use a minimum of $N_{e}=8$ elements per wavelength results in a finite element size $l_{e}=0.75 \mathrm{~m}$. It will be demonstrated in a following subsection, however, that the application of this rule of thumb to the boundary element mesh along the soil-structure interface results in a smaller boundary element size $l_{e}=0.50 \mathrm{~m}$. In order to ensure compatibility of both finite element and boundary element meshes, a finite element size $l_{e}=0.50 \mathrm{~m}$ is therefore used throughout the structure, corresponding to a larger number of elements per wavelength $\left(N_{e}=12\right)$.

\subsection{Kinematics of the structure}

The modes $\boldsymbol{\Psi}_{b_{1}}$ of the superstructure clamped at the base are dominated by bending modes of the floors and the walls. Figure 9 shows the first mode at $11.58 \mathrm{~Hz}$ for the structure on a strip foundation, clamped along the interface between the superstructure and the foundation, revealing bending of the slabs on the first and the second floor. In-plane deformations of the (stiff) structural walls are only observed in mode shapes corresponding to eigenfrequencies far beyond the frequency range of excitation, that are unlikely to contribute to the structural response.

The modes $\boldsymbol{\Psi}_{b_{1} j}(j=1, \ldots, 6)$ of the free foundation correspond to rigid body translation in the $x$-, $y$ - and $z$-directions and rigid body rotation modes around the $x$-axis, the $y$-axis (rocking modes), and the $z$-axis (torsional mode). The scaling of these modes is such that they correspond to a unit displacement or rotation, allowing for a quantitative comparison of the corresponding elements of the soil impedance matrix and the modal load vector. The flexible foundation modes $\boldsymbol{\Psi}_{b_{1} j}(j=7, \ldots, l)$, on the other hand, are scaled with respect to the mass matrix $\mathbf{M}_{b_{2} b_{2}}^{\prime}$ of the free foundation, so that $\boldsymbol{\Psi}_{b_{1} j}^{\mathrm{T}} \mathbf{M}_{b_{2} b_{2}}^{\prime} \boldsymbol{\Psi}_{b_{1} j}=1$.

The static transmission of the free foundation modes into the structure is illustrated in figure 10. Whereas the static transmission of the rigid body foundation modes does not deform the structure, the flexible foundation modes induce a global deformation of the superstructure corresponding to a large strain energy. Therefore, the modal contribution of the flexible foundation modes is expected to be small with respect that of the rigid foundation modes and the modes of the superstructure clamped at the base.

\subsection{Soil impedance}

A boundary element method based on the Green's functions of a linear elastic halfspace is applied for the calculation of the soil impedance matrix $\boldsymbol{\Psi}_{b_{2}}^{\mathrm{T}} \hat{\mathbf{K}}_{b_{2} b_{2}}^{s}(\omega) \boldsymbol{\Psi}_{b_{2}}$ and the load vector $\boldsymbol{\Psi}_{b_{2}}^{\mathrm{T}} \hat{\mathbf{f}}_{b_{2}}(\omega)$ defined in equations (19) and (20). Therefore, the soil-structure interface $\Sigma_{b s}$ is modelled with 4-node quadrilateral boundary elements, using elementwise constant interpolation functions for displacements and tractions. The minimum wavelength in the soil is equal to $\lambda_{\min }=C_{s} / f_{\max }=4 \mathrm{~m}$ at the upper bound $f_{\max }=50 \mathrm{~Hz}$ of the frequency range of interest. $N_{e}=8$ boundary elements are used per wavelength, resulting in a boundary element size $l_{e}=0.5 \mathrm{~m}$, which is compatible with the finite element size of the structure's foundation.

Using a boundary element formulation to compute the impedance of an embedded foundation may result in fictitious eigenfrequencies at the eigenfrequencies of the bounded interior soil domain $\Omega_{s}^{\text {int }}$ with Dirichlet boundary conditions along the soil-structure interface $\Sigma_{b s}$ and free surface conditions on the free surface $[5,11]$. In order to mitigate this problem, an alternative boundary element formulation is used, that is based on a linear combination of the boundary integral equations in terms of displacements and normal displacement derivatives, using a complex coupling parameter [26].

The real part of the first six diagonal elements of the dynamic soil impedance matrix $\boldsymbol{\Psi}_{b_{2}}^{\mathrm{T}} \hat{\mathbf{K}}_{b_{2} b_{2}}^{s}(\omega) \boldsymbol{\Psi}_{b_{2}}$ is plotted in figure 11 for the structure with a slab, a strip and a box foundation. The first three diagonal elements correspond to a rigid translation of the foundation in the $x$-, $y$ - and $z$-direction, while the following three elements are related to a rotation around the $x-, y$ - (rocking) and $z$-axis (torsion) of the Cartesian frame of reference, respectively. These impedance coefficients represent the forces needed to generate the corresponding unit rigid body displacement of the foundation, allowing to easily compare results obtained for different foundation types.

For all modes, the dynamic soil stiffness of the box foundation is larger than the corresponding dynamic soil stiffness of the slab foundation. This embedment effect [21] is less pronounced for the vertical translation 
mode (figure 11c), whereas a factor of two is observed between the horizontal stiffnesses (figures 11a and $11 \mathrm{~b})$ and the rocking and torsional stiffness (figures $11 \mathrm{~d}$ to $11 \mathrm{f}$ ).

For the strip foundation, the impedance is similar as in the case of the slab foundation for frequencies up to $5 \mathrm{~Hz}$. A large increase in stiffness is observed for higher frequencies, however, which is caused by an anti-resonance effect of the soil enclosed by the foundation strips. Figure 12 shows the wave field scattered in the soil by the vertical rigid body mode of the strip foundation at $5 \mathrm{~Hz}$ and at $20 \mathrm{~Hz}$. Whereas a uniform displacement field is observed at $5 \mathrm{~Hz}$, the anti-phase resonance of the soil between the strips at $20 \mathrm{~Hz}$ causes large strains at the foundation toe. The corresponding soil stresses results in an increased dynamic soil stiffness.

\subsection{Modal load vector}

The modal load vector $\boldsymbol{\Psi}_{b_{2}}^{\mathrm{T}} \hat{\mathbf{f}}_{b_{2}}(\omega)$, defined in equation (20), differs for the three foundation types under consideration, as shown in figure 13. A large modal load vector can compensate the decrease of the structural response due to a larger dynamic soil stiffness when comparing two types of foundation.

For all foundation types, the vertical rigid body translation mode and the rocking mode around the $y$-axis are mainly excited by the incident wave field, that is dominated by Rayleigh waves, resulting in a large amplitude of the corresponding elements of the modal load vector.

For the surface slab foundation, the tractions $\hat{\boldsymbol{t}}_{s}\left(\boldsymbol{u}_{\text {inc }}\right)$ on the soil-structure interface $\Sigma_{b s}$ due to the incident wave field $\boldsymbol{u}_{\text {inc }}$ vanish. The modal load vector reduces to the integral $-\int_{\Sigma_{b s}}\left(\boldsymbol{\Psi}_{b_{2}} \mathbf{N}_{b_{2}}\right)^{\mathrm{T}} \hat{\boldsymbol{t}}_{s}\left(\boldsymbol{u}_{\mathrm{d} 0}\right) d \Sigma$ of the tractions $\hat{\boldsymbol{t}}_{s}\left(\boldsymbol{u}_{\mathrm{d} 0}\right)$ due to the locally diffracted wave field $\boldsymbol{u}_{\mathrm{d} 0}$. The amplitude of all components of the modal load vector related to rigid body foundation modes is larger for the box foundation than for the slab foundation since (a) the soil tractions $\hat{\boldsymbol{t}}_{s}\left(\boldsymbol{u}_{\text {inc }}\right)$ on the embedded soil-structure interface $\Sigma_{b s}$ are not vanishing, (b) the area of the box foundation is larger than the slab foundation due to the presence of the foundation's side walls, and (c) the embedment of the foundation results in larger tractions $\hat{\boldsymbol{t}}_{s}\left(\boldsymbol{u}_{\mathrm{d} 0}\right)$ associated with the locally diffracted wave field $\boldsymbol{u}_{\mathrm{d} 0}$.

While the total area of the strip foundation is smaller than the area of the slab foundation, the non-zero soil tractions $\hat{\boldsymbol{t}}_{s}\left(\boldsymbol{u}_{\text {inc }}\right)$ and the larger tractions $\hat{\boldsymbol{t}}_{s}\left(\boldsymbol{u}_{\mathrm{d} 0}\right)$ result in a larger amplitude of all components of the modal load vector for the strip foundation.

\subsection{Convergence analysis}

The calculation of the structural response is based on the decomposition (14) of the displacement vector into modes clamped at the base and the quasi-static transmission of free foundation modes. A sufficient number of modes should be taken into account in order to obtain accurate results. Convergence is studied using the displacement norm $\left\|\mathbf{u}_{b}\right\|_{B}$, defined as the root mean square of the norm of the structural displacement vector in the frequency range of interest $B=\left[0, \omega_{\max }\right]$, where $\omega_{\max }=2 \pi 50 \mathrm{rad} / \mathrm{s}$ :

$$
\left\|\mathbf{u}_{b}\right\|_{B}=\sqrt{\frac{1}{\omega_{\max }} \int_{0}^{\omega_{\max }}\left\|\hat{\mathbf{u}}_{b}(\omega)\right\|^{2} d \omega}
$$

Figure 14 shows the displacement norm $\left\|\mathbf{u}_{b}\right\|_{B}$ for the structure with a slab foundation, a strip and a box foundation as a function of the number $l$ of modes of the superstructure clamped at the base and the number $k$ of free foundation modes, as employed in the decomposition (14) of the structural displacement vector $\hat{\mathbf{u}}_{b}(\omega)$. The displacement norm $\left\|\mathbf{u}_{b}\right\|_{B}$ has converged satisfactorily when 20 free foundation modes and 20 modes of the superstructure clamped at the base are used, which is the number of modes used in all subsequent calculations.

\section{Influence of the foundation type on the structural response}

Considering the response of the structure during the passage of the truck on the traffic plateau, it is instructive to first compare the incident wave field and the predicted response at the soil-foundation interface. 
The solid line on figure 15 shows the vertical displacements along the edge AB between the foundation and the structure at time $t=-0.37 \mathrm{~s}$. In the three cases considered, the predicted displacements along the edge AB clearly differ from the incident wave field, indicated with crosses, that would occur before excavation of the soil. The response turns out to be stiffer, as the global building motion is dominated by the large in-plane stiffness of the structural walls and the rigid body kinematics of the foundation. In the case of a structure with a slab foundation, the structural walls stiffen the slab along its edges (while it may still deform in the middle); the stiffness of the strip and box foundation is higher than the slab foundation, resulting in an even stiffer response along the edge AB. For the three cases considered, in-plane wall deformations occur in the higher structural modes, which are not expected to have an important contribution to the structural response, given the low frequency content of the excitation. The previous observations are consistent with the general understanding that the global motion of a relatively stiff building resting on a soft soil is dominated by rigid body kinematics with little deformation of the walls occurs, whereas a structure supported by a stiff soil undergoes quasi-static wall deformations, following the ground motion.

In the following, the influence of the foundation embedment depth on the structural response in different points in the structure is studied, primarily concentrating on the rigid body foundation modes.

Figure 16 shows the time history and the frequency content of the horizontal velocity $v_{b x}$ in the point I on the edge between the front wall and the foundation (figure 4) for the structure with a slab, a strip and a box foundation. For the structure with a box foundation, the velocity is two times smaller than for the structure with a strip foundation, due to the large embedment effect on the impedance corresponding to the horizontal foundation displacement modes (figure 11a and b). The higher mass of the box foundation contributes to this decrease, certainly at high frequencies. A similar effect is observed for the structure with a strip foundation. The response in the point I significantly differs from the incident wave field, which indicates the relative importance of dynamic soil-structure interaction effects.

The horizontal velocity $v_{b x}$ in the point $\mathrm{M}$ (figure 17) on the edge between the front wall and the slab on the second floor (figure 4) is larger than the corresponding velocity in the point I on the edge between the front wall and the foundation (figure 16) for all foundation types, illustrating the contribution of the rocking mode of the foundation around the $y$-axis. In the case of a box foundation, the reduction of the impedance coefficient of this rocking mode due to the embedment (figure 11e) is compensated by the a large modal load (figure 13e), resulting in a larger horizontal velocity than for the structure with a slab or strip foundation.

The time history and frequency content of the vertical velocity $v_{b z}$ in the point I (figure 18) reveal a relatively small embedment effect for the structure with a box and a strip foundation. Due to dynamic soil-structure interaction, the peak particle velocity of the structural response in this point is smaller than the incident wave field.

Contrary, the response in the point K (figure 19) on the edge between the rear wall and the foundation (figure 4) is larger than the incident wave field. The relatively stiff structural response results in a large contribution of the rocking foundation mode, affecting the wave fronts travelling from the front (point I) to the back (point $\mathrm{K}$ ) of the building. The vertical velocity in the point $\mathrm{M}$ on the edge between the front wall and the slab on the second floor is very similar as in the point I due to the high structural stiffness in the vertical direction.

Figure 20 shows the time history and the frequency content of the vertical velocity in the point $\mathrm{L}$ in the centre of the slab on the first floor (figure 4) for the structure with the box foundation (very similar results are obtained for the structure with the slab and the strip foundation). The frequency content is dominated by a peak at $11.58 \mathrm{~Hz}$, corresponding to the first eigenmode of the slab. A modal damping ratio $\xi=0.0325$ can be estimated from the logarithmic decrement of the response in this point, which is larger than the applied modal damping ratio $\xi=0.02$, since dynamic soil-structure interaction increases the attenuation due to geometric and material damping in the soil. This increased attenuation is also observed in the time history of the vertical velocity in the points I and K (figures 18 and 19). In the time period between $t=1.2 \mathrm{~s}$ and $t=2 \mathrm{~s}$, a small oscillation with a period of $0.1 \mathrm{~s}(f=10 \mathrm{~Hz})$ is observed, illustrating the dissipation of energy in the soil.

The resulting effect of the foundation type on the structural response is different for the horizontal and vertical directions. The influence of the foundation type on the vertical structural response is small, as the decrease in the response due to the foundation embedment is compensated by an increased modal load 
vector. A substantial difference between the horizontal structural response for different foundation types is observed due to the large effect of foundation embedment, which is not compensated by a large modal load vector effect.

\section{The importance of dynamic soil-structure interaction}

The calculation of the structural response by means of equation (18) necessitates the determination of the dynamic stiffness matrix of the soil $\boldsymbol{\Psi}_{b_{2}}^{\mathrm{T}} \hat{\mathbf{K}}_{b_{2} b_{2}}^{s}(\omega) \boldsymbol{\Psi}_{b_{2}}$ and the equivalent load vector $\boldsymbol{\Psi}_{b_{2}}^{\mathrm{T}} \hat{\mathbf{f}}_{b_{2}}(\omega)$ by means of a boundary element formulation, which is computationally expensive. The computational cost can be reduced using a simplified method of analysis that neglects dynamic soil-structure interaction effects but accounts for the relative stiffness of the structure and the soil. In this section, such an approximate method, referred to as a n-SSI calculation, is compared with a method that fully accounts for dynamic soil-structure interaction.

In the SSI calculation, equation (18) is rigorously solved. For the n-SSI-calculation, a technique is proposed where the projection $\left.\hat{\mathbf{u}}_{b_{2}}(\omega)\right|_{\Sigma_{b s}}$ of the foundation displacement $\hat{\mathbf{u}}_{b_{2}}(\omega)$ on the soil-structure interface $\Sigma_{b s}$ is written as a linear combination of the projections $\left.\boldsymbol{\Psi}_{b_{2}}\right|_{\Sigma_{b s}}$ of foundation modes $\boldsymbol{\Psi}_{b_{2}}$ on the interface $\Sigma_{b s}$ :

$$
\left.\hat{\mathbf{u}}_{b_{2}}(\omega)\right|_{\Sigma_{b s}}=\left.\boldsymbol{\Psi}_{b_{2}}\right|_{\Sigma_{b s}} \hat{\boldsymbol{\alpha}}_{b_{2}}(\omega)
$$

The displacement field $\left.\hat{\mathbf{u}}_{b_{2}}(\omega)\right|_{\Sigma_{b s}}$ is an approximation of the incident wave field $\hat{\mathbf{u}}_{\text {inc }}(\omega)$ :

$$
\left.\hat{\mathbf{u}}_{b_{2}}(\omega)\right|_{\Sigma_{b s}} \simeq \hat{\mathbf{u}}_{\text {inc }}(\omega)
$$

The modal coordinates $\hat{\boldsymbol{\alpha}}_{b_{2}}(\omega)$ in equation (23) are found as the solution of the following least squares problem:

$$
\hat{\boldsymbol{\alpha}}_{b_{2}}(\omega)=\left.\left(\left.\left.\boldsymbol{\Psi}_{b_{2}}\right|_{\Sigma_{b s}} ^{\mathrm{T}} \boldsymbol{\Psi}_{b_{2}}\right|_{\Sigma_{b s}}\right)^{-1} \boldsymbol{\Psi}_{b_{2}}\right|_{\Sigma_{b s}} ^{\mathrm{T}} \hat{\mathbf{u}}_{\mathrm{inc}}(\omega)
$$

If the soil is rigid with respect to the structure, the foundation displacements are similar to the incident wave field. The number of foundation modes $\left.\boldsymbol{\Psi}_{b_{2}}\right|_{\Sigma_{b s}}$ should be sufficiently large to represent the flexible behaviour of the soil-structure interface. The response can be computed using the same number of foundation modes as in the SSI calculation, resulting in a good correspondance between the incident wave field and the foundation displacement. This calculation is referred to as the n-SSI-FF calculation.

If the structure is more rigid than the soil, the kinematics of the foundation are restricted to its rigid body modes (n-SSI-RF calculation). The least squares problem (25) then consists of finding the modal coordinates $\hat{\boldsymbol{\alpha}}_{b_{1}}(\omega)$ of the rigid body modes that result in the best spatial average of the incident wave field.

The first of equations (18) allows to calculate the modal coordinates $\hat{\boldsymbol{\alpha}}_{b_{2}}(\omega)$ from the modal coordinates $\hat{\boldsymbol{\alpha}}_{b_{1}}(\omega)$ :

$$
\left(\boldsymbol{\Lambda}_{k k}-\omega^{2} \mathbf{I}_{k k}\right) \hat{\boldsymbol{\alpha}}_{b_{1}}(\omega)=\omega^{2}\left(\boldsymbol{\Psi}_{b_{1}}^{\mathrm{T}} \mathbf{M}_{b_{1} b_{1}} \boldsymbol{\Psi}_{b_{1}}^{s}+\boldsymbol{\Psi}_{b_{1}}^{\mathrm{T}} \mathbf{M}_{b_{1} b_{2}} \boldsymbol{\Psi}_{b_{2}}\right) \hat{\boldsymbol{\alpha}}_{b_{2}}(\omega)
$$

The structural displacement vector is finally obtained by means of equation (14).

The difference between the results of the SSI, the n-SSI-FF and the n-SSI-RF calculation is investigated considering the vertical displacement along the edge AB between the structure and the foundation (figure 4) at time $t=-0.37 \mathrm{~s}$, as shown on figure 15 . The $\mathrm{n}-\mathrm{SSI}-\mathrm{RF}$ calculation results in an almost linear deformation along the edge $\mathrm{AB}$ of the foundation, which is a better approximation of the structural response revealed by the SSI calculation than the approximation obtained with the n-SSI-FF calculation. In the latter case, the displacements on the edge $\mathrm{AB}$ between the structure and the foundation are close to the incident wave field, which induces excessive and unrealistic wall deformations.

In the case of a structure resting on a stiff soil, which is not considered in the present paper, the walls would undergo quasi-static deformation following the ground motion. In this case, dynamic soil-structure interaction effects are negligible and the n-SSI-FF calculation will result in a good approximation of the structural response. This has important consequences for vibration induced damage to buildings: wall cracking, caused by excessive deformations, is more likely in the case of a quasi-static transmission of the ground motion into the walls. 


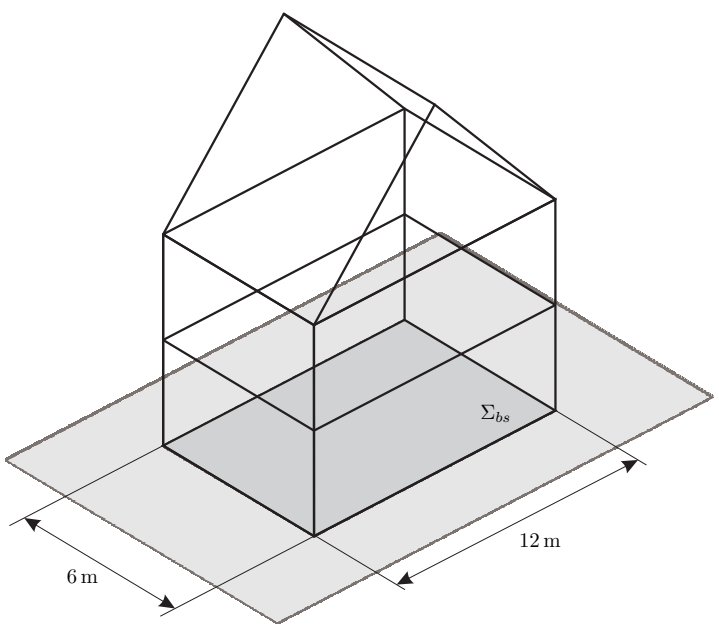

a. Slab foundation.

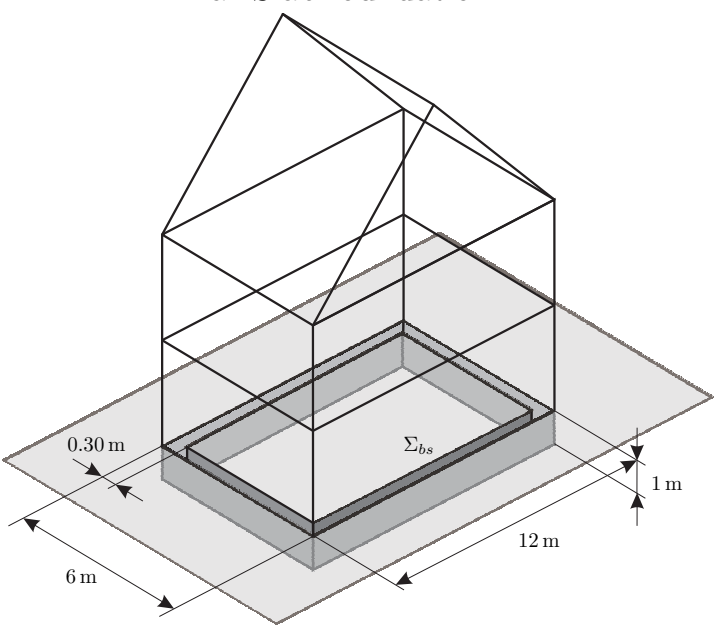

b. Strip foundation.

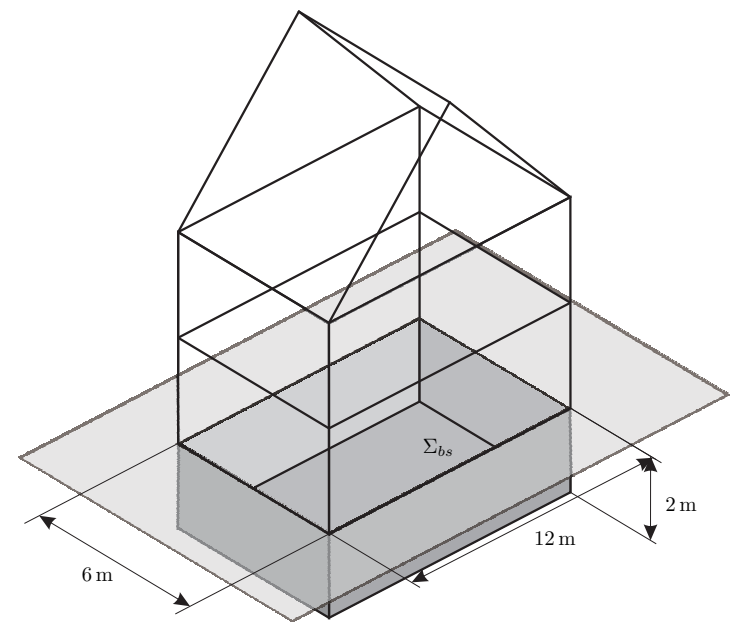

c. Box foundation.

Figure 8: Geometry of the (1) slab, (2) strip and (3) box foundation. The dark grey surface represents the soil-structure interface $\Sigma_{b s}$. 


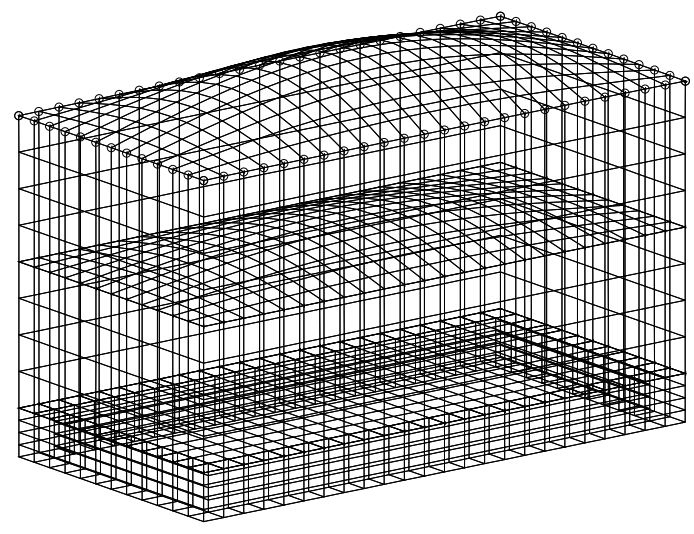

Figure 9: First mode at $11.58 \mathrm{~Hz}$ of the superstructure clamped at the base for the structure with a strip foundation 


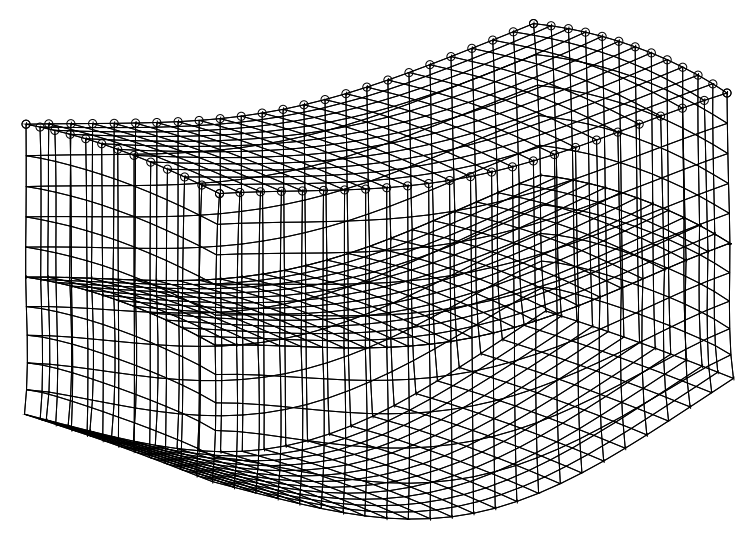

a. Mode 7 at $7.84 \mathrm{~Hz}$.

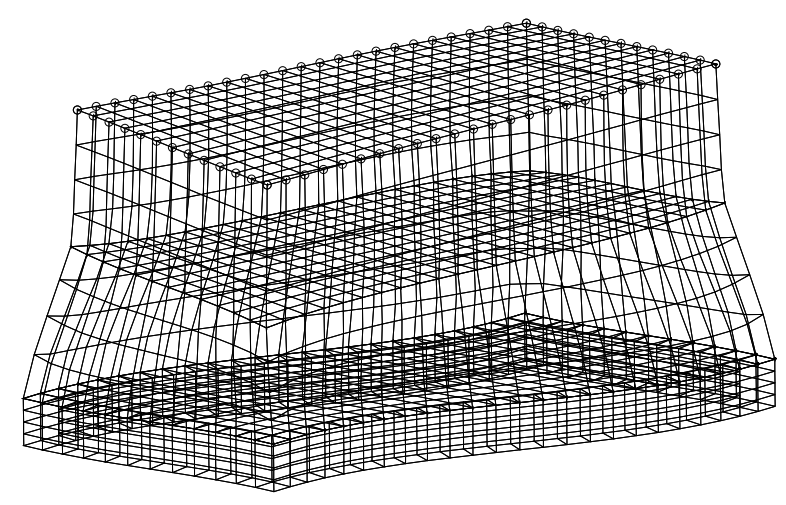

b. Mode 7 at $55.93 \mathrm{~Hz}$.

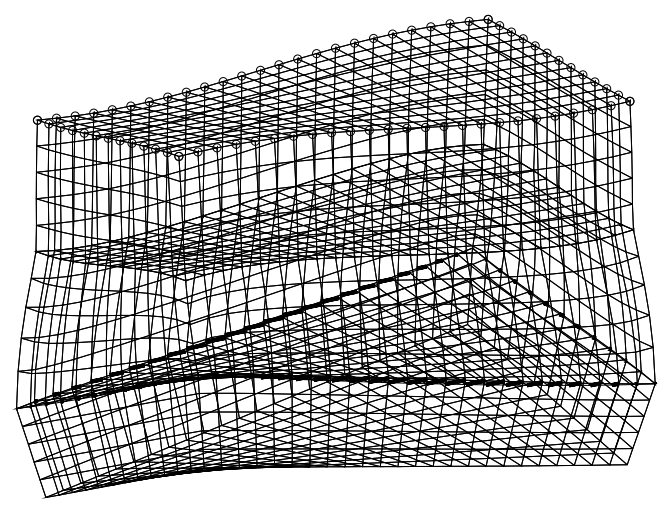

c. Mode 7 at $48.47 \mathrm{~Hz}$.

Figure 10: Quasi-static transmission of the seventh foundation mode for the structure with (a) a slab, (b) a strip and (c) a box foundation. 


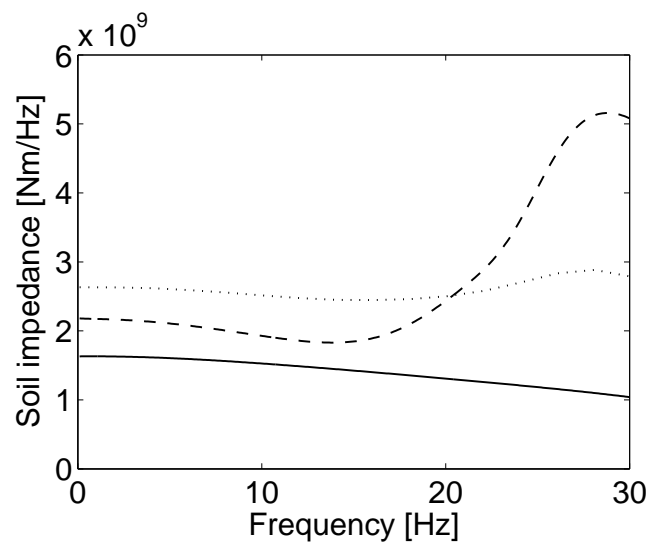

a. $\operatorname{Re}\left[\boldsymbol{\Psi}_{b_{2}}^{\mathrm{T}} \hat{\mathbf{K}}_{b_{2} b_{2}}^{s}(\omega) \boldsymbol{\Psi}_{b_{2}}(1,1)\right]$.

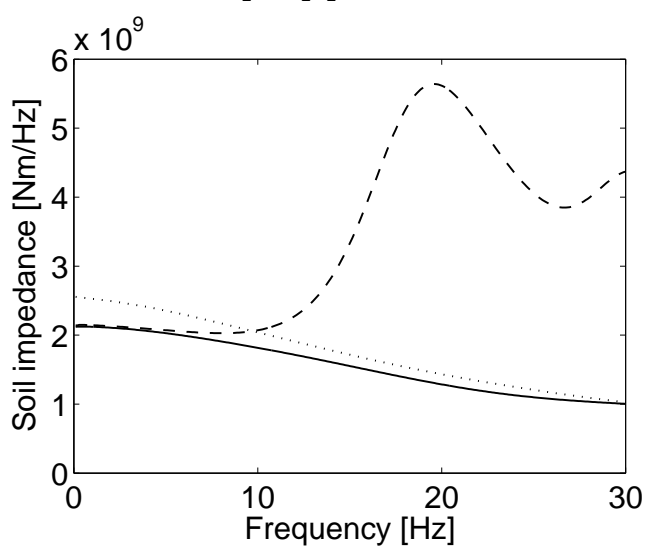

c. $\operatorname{Re}\left[\boldsymbol{\Psi}_{b_{2}}^{\mathrm{T}} \hat{\mathbf{K}}_{b_{2} b_{2}}^{s}(\omega) \boldsymbol{\Psi}_{b_{2}}(3,3)\right]$.

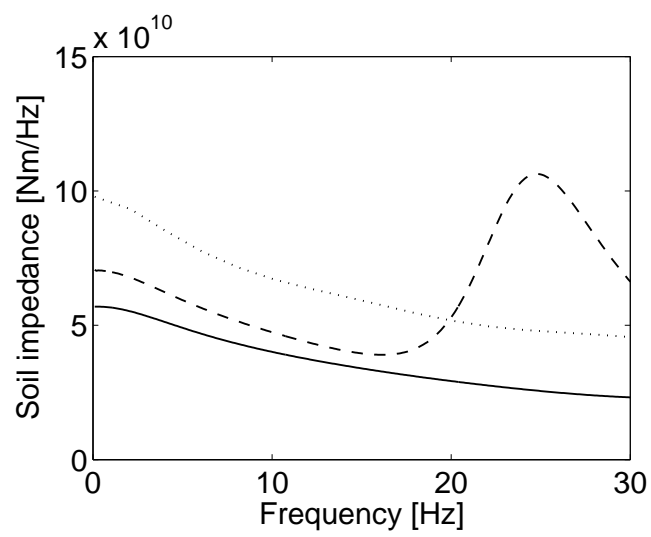

e. $\operatorname{Re}\left[\boldsymbol{\Psi}_{b_{2}}^{\mathrm{T}} \hat{\mathbf{K}}_{b_{2} b_{2}}^{s}(\omega) \boldsymbol{\Psi}_{b_{2}}(5,5)\right]$.

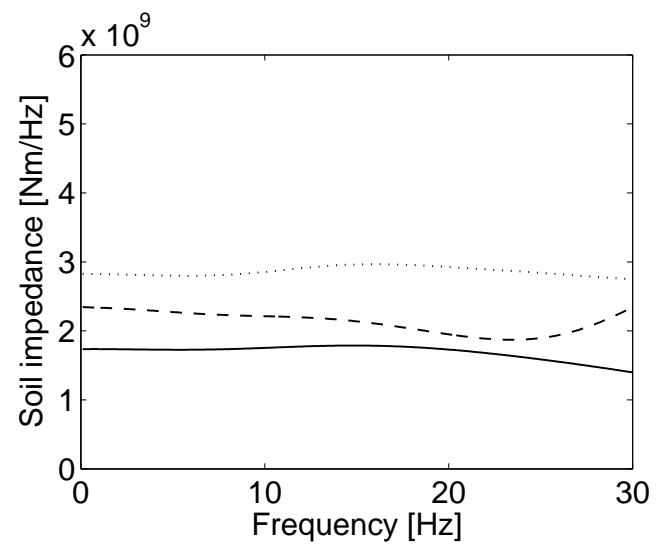

b. $\operatorname{Re}\left[\boldsymbol{\Psi}_{b_{2}}^{\mathrm{T}} \hat{\mathbf{K}}_{b_{2} b_{2}}^{s}(\omega) \boldsymbol{\Psi}_{b_{2}}(2,2)\right]$.

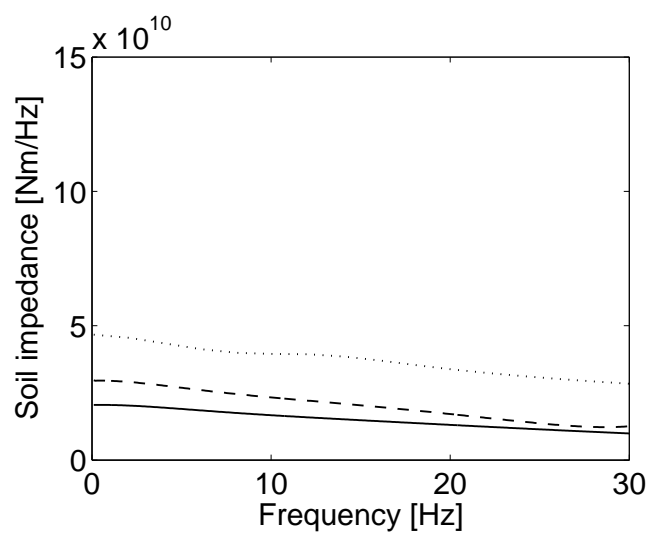

d. $\operatorname{Re}\left[\boldsymbol{\Psi}_{b_{2}}^{\mathrm{T}} \hat{\mathbf{K}}_{b_{2} b_{2}}^{s}(\omega) \boldsymbol{\Psi}_{b_{2}}(4,4)\right]$.

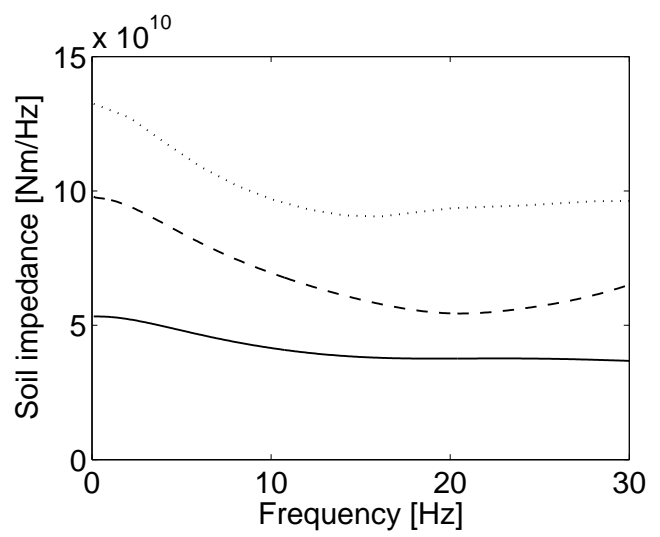

f. $\operatorname{Re}\left[\boldsymbol{\Psi}_{b_{2}}^{\mathrm{T}} \hat{\mathbf{K}}_{b_{2} b_{2}}^{s}(\omega) \boldsymbol{\Psi}_{b_{2}}(6,6)\right]$.

Figure 11: Real part of the first six diagonal elements of the soil impedance matrix $\boldsymbol{\Psi}_{b_{2}}^{\mathrm{T}} \hat{\mathbf{K}}_{b_{2} b_{2}}^{s}(\omega) \boldsymbol{\Psi}_{b_{2}}$ for the structure with a slab (solid line), a strip (dashed line) and a box (dotted line) foundation. 

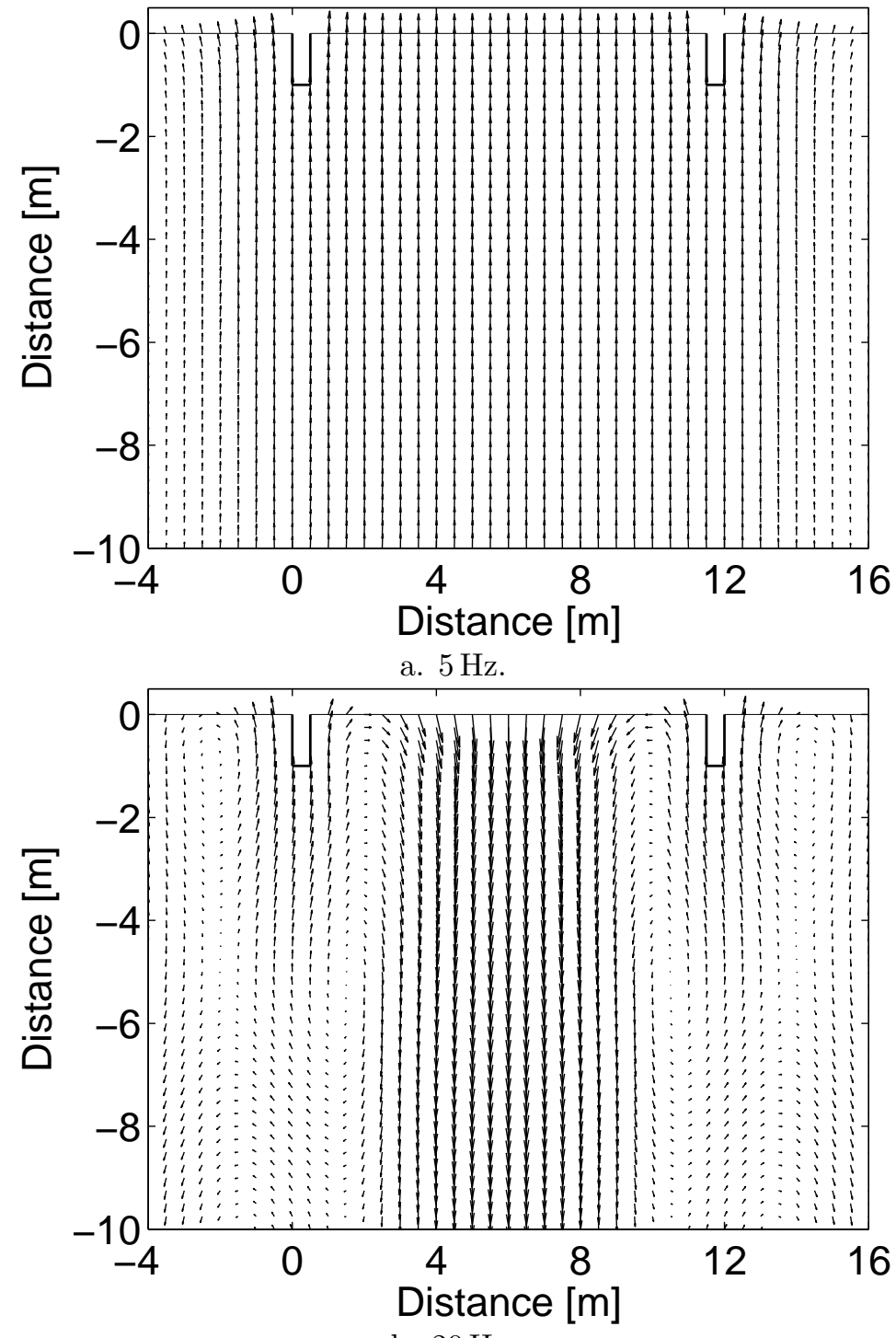

b. $20 \mathrm{~Hz}$.

Figure 12: Wave field scattered by the vertical rigid body mode of the strip foundation in the plane $y=3 \mathrm{~m}$ at (a) $5 \mathrm{~Hz}$ and (b) $20 \mathrm{~Hz}$. 

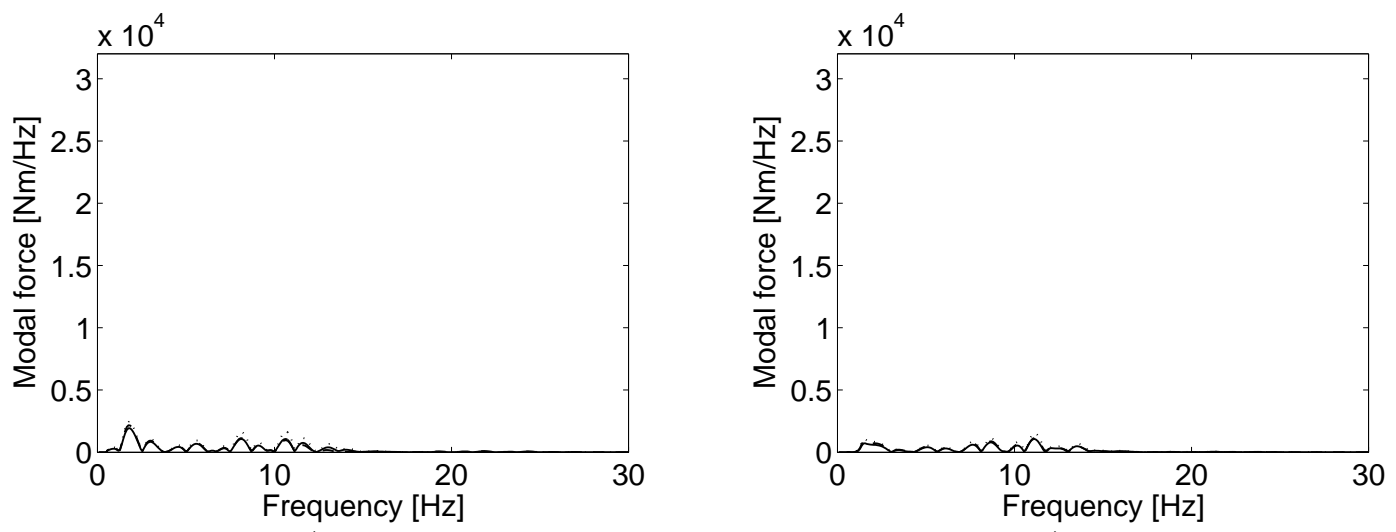

a. $\left|\boldsymbol{\Psi}_{b_{2}}^{\mathrm{T}} \hat{\mathbf{f}}_{b_{2}}(\omega)(1)\right|$.

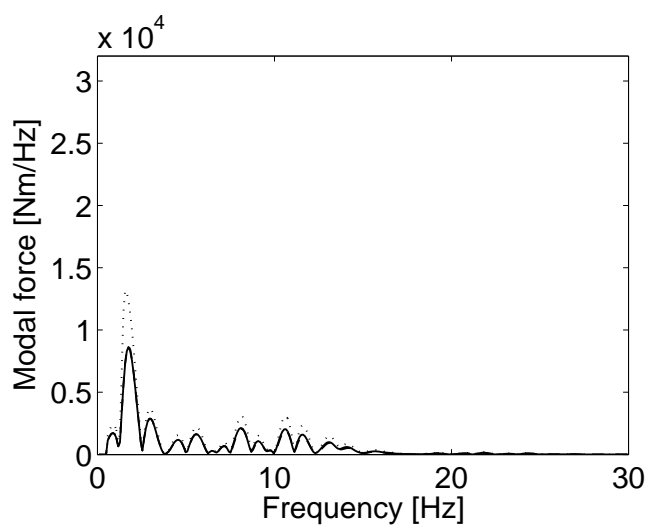

b. $\left|\boldsymbol{\Psi}_{b_{2}}^{\mathrm{T}} \hat{\mathbf{f}}_{b_{2}}(\omega)(2)\right|$.

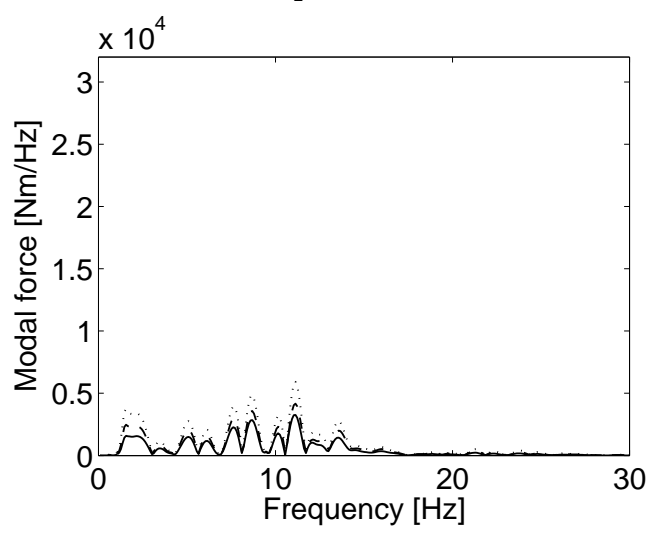

c. $\left|\boldsymbol{\Psi}_{b_{2}}^{\mathrm{T}} \hat{\mathbf{f}}_{b_{2}}(\omega)(3)\right|$.
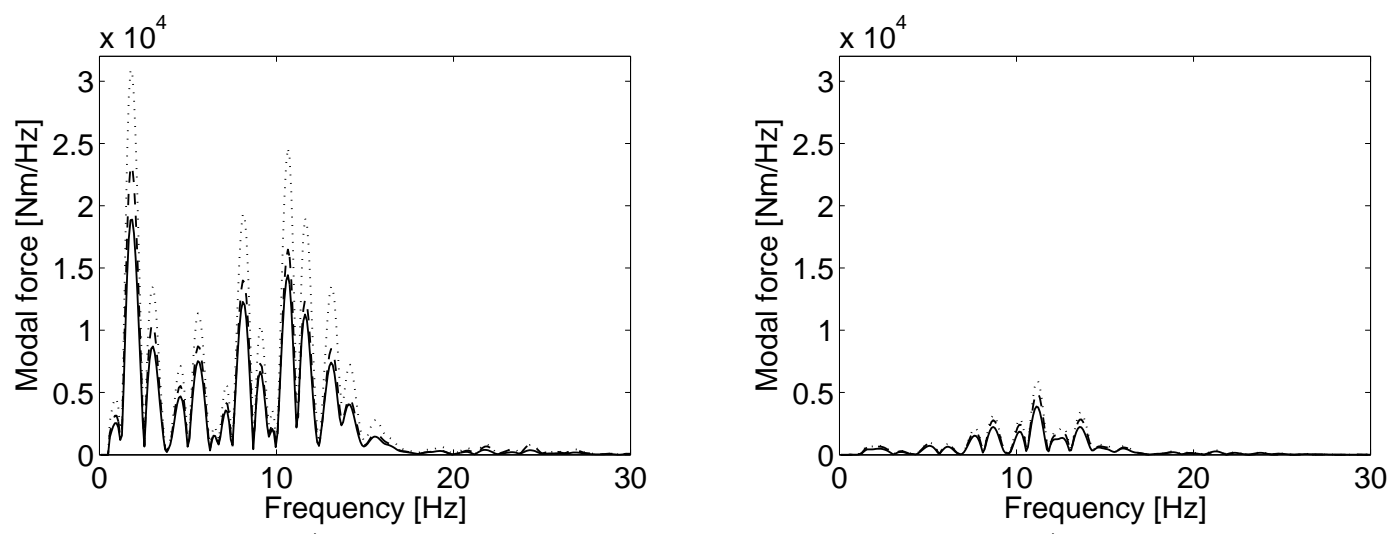

e. $\left|\boldsymbol{\Psi}_{b_{2}}^{\mathrm{T}} \hat{\mathbf{f}}_{b_{2}}(\omega)(5)\right|$.

f. $\left|\boldsymbol{\Psi}_{b_{2}}^{\mathrm{T}} \hat{\mathbf{f}}_{b_{2}}(\omega)(6)\right|$.

Figure 13: Modulus of first six elements of the modal load vector $\Psi_{b_{2}}^{\mathrm{T}} \hat{\mathbf{f}}_{b_{2}}(\omega)$ for the structure with a slab (solid line), a strip (dashed line) and a box (dotted line) foundation. 


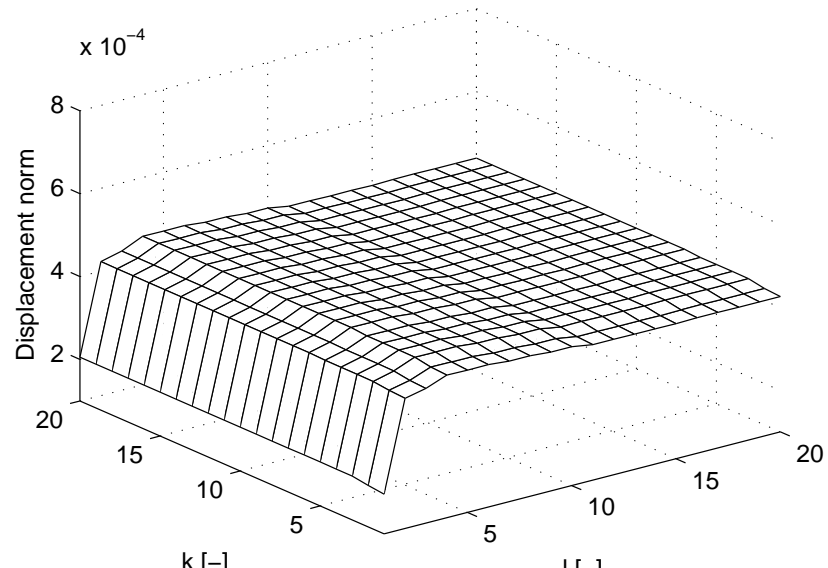

a. Slab foundation.

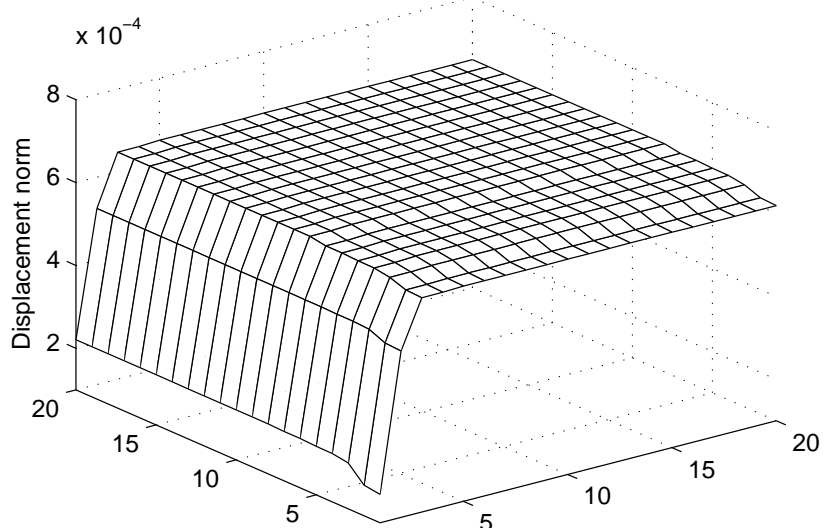

$\mathrm{k}[-]$

b. Strip foundation.

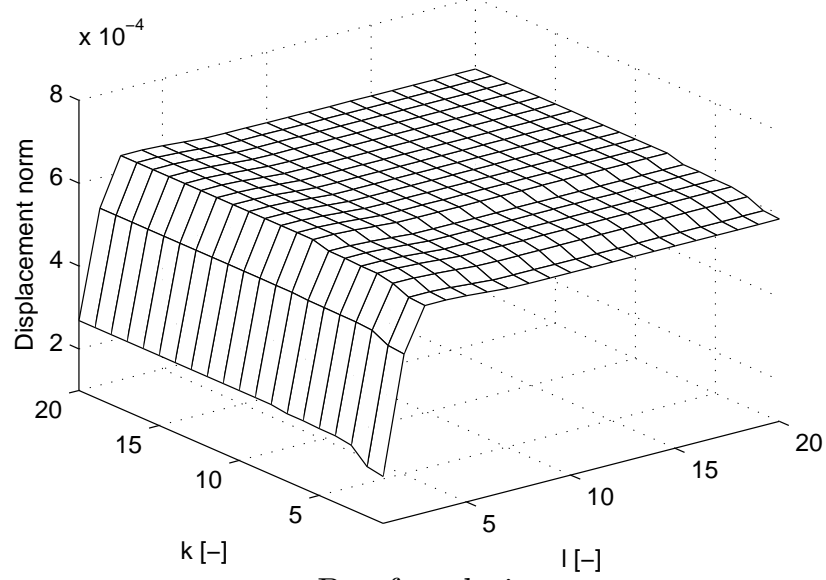

c. Box foundation.

Figure 14: Displacement norm $\left\|\mathbf{u}_{b}\right\|_{B}$ as a function of the number $l$ of modes of the superstructure clamped at the base and the number $k$ of free foundation modes for the structure with (a) a slab, (b) a strip and (c) a box foundation. 


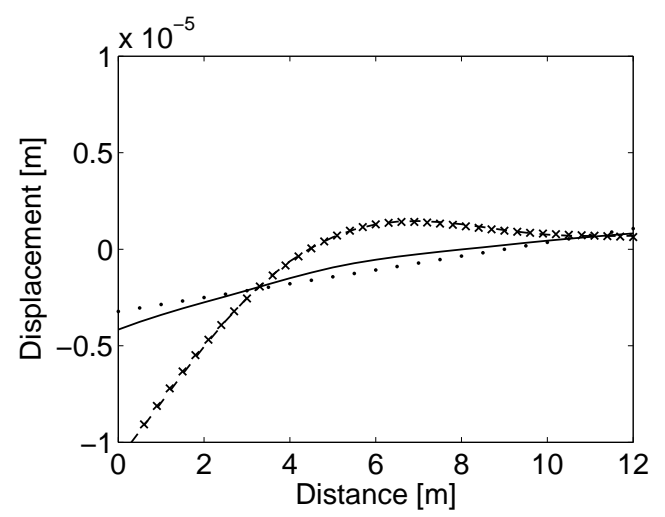

a. Slab foundation.

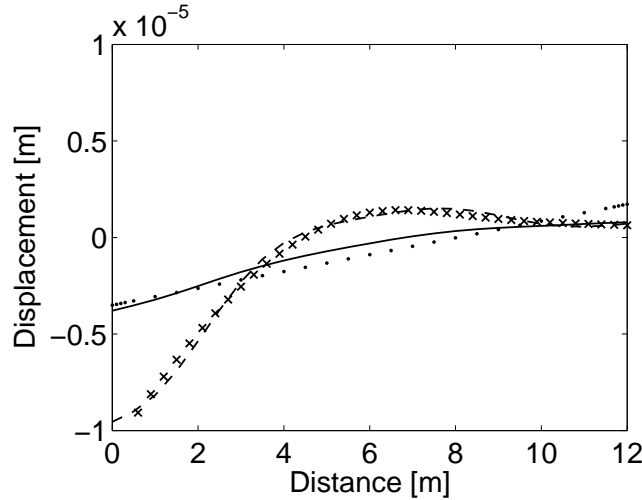

b. Strip foundation.

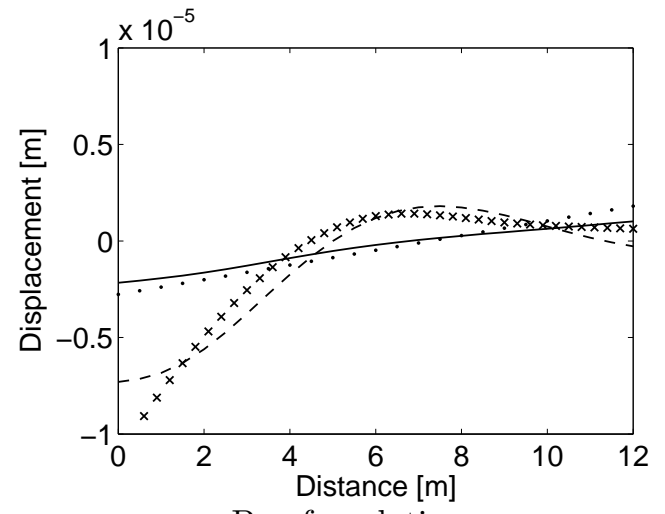

c. Box foundation.

Figure 15: Vertical displacement of the edge $\mathrm{AB}$ between the foundation and the structure at time $t=-0.37 \mathrm{~s}$ for the structure with (a) a slab, (b) a strip and (c) a box foundation. Results are shown for a calculation that accounts for dynamic soilstructure interaction (solid line), the n-SSI-FF (dashed line) and the n-SSI-RF (dotted line) calculation. The incident wave field is indicated with crosses. 
1.
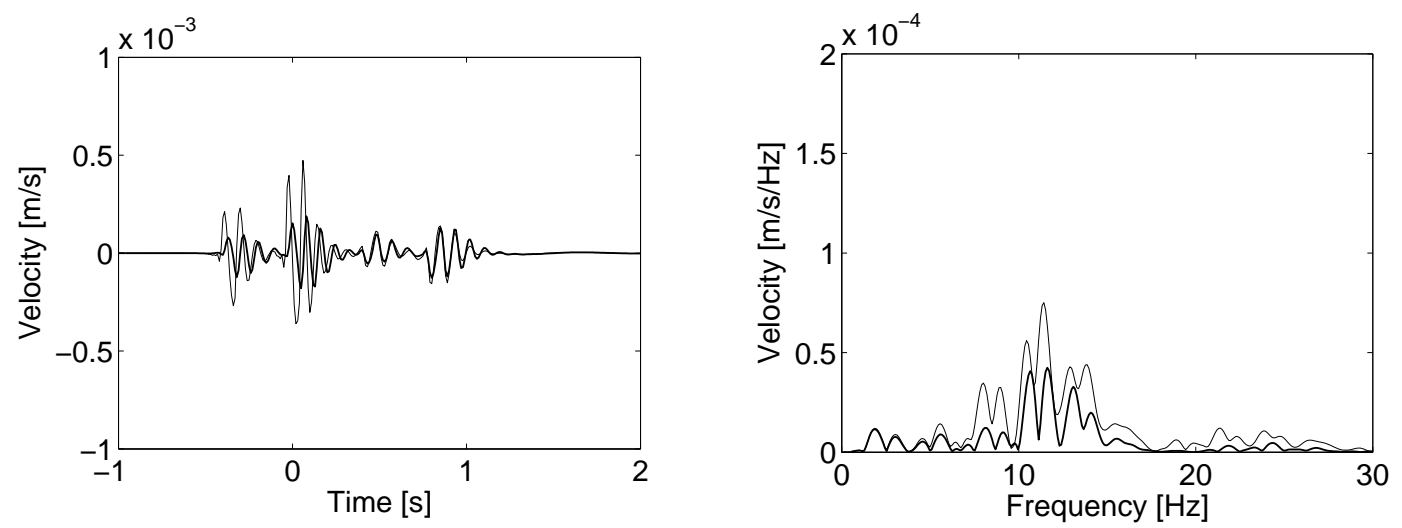

2.
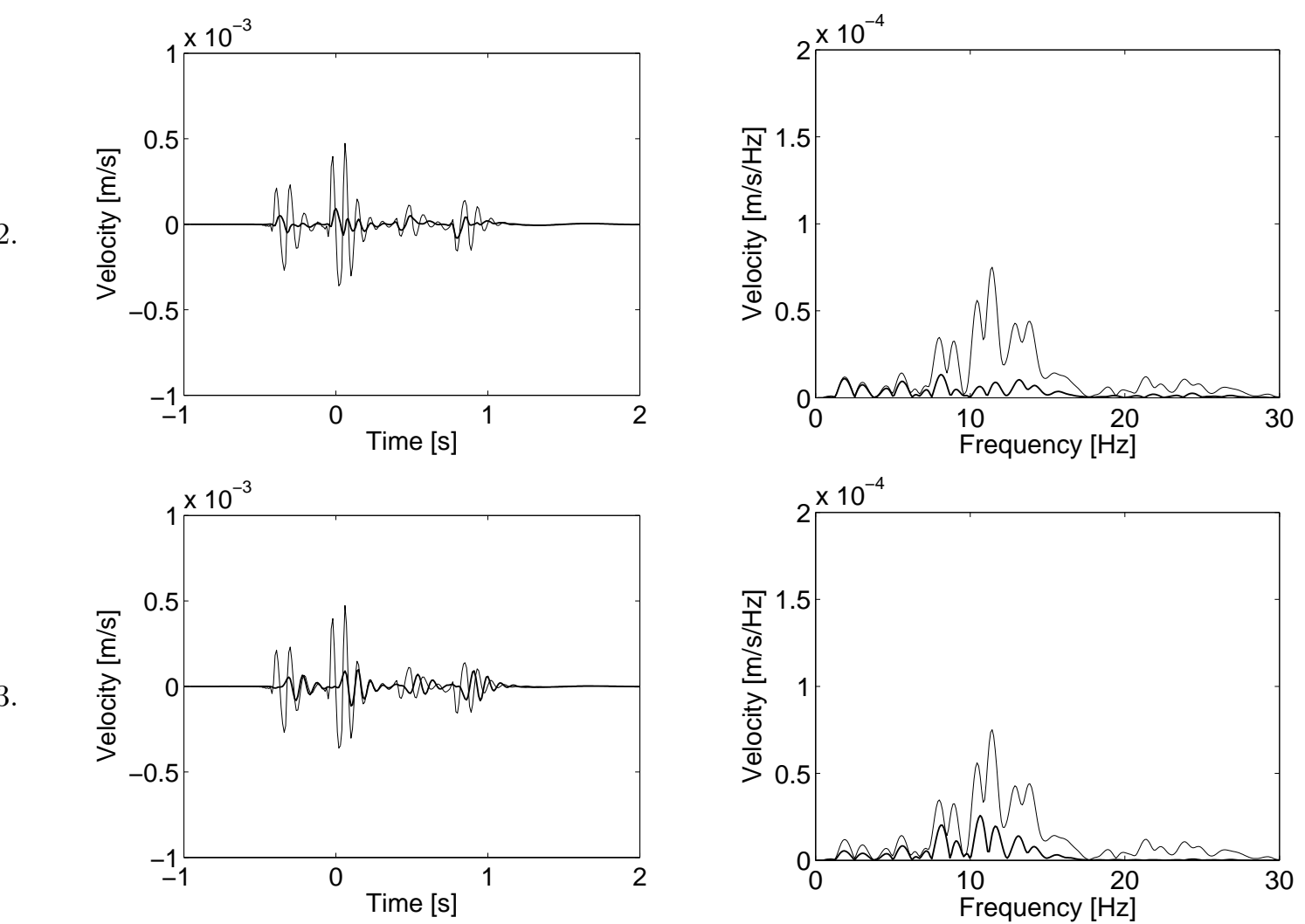

a. $v_{b x}(t)$.

b. $\hat{v}_{b x}(\omega)$.

Figure 16: (a) Time history and (b) frequency content of the horizontal velocity in the point I on the edge between the front wall and the foundation for the structure with (1) a slab, (2) a strip and (3) a box foundation. The incident wave field is indicated with a thin line. 
1.
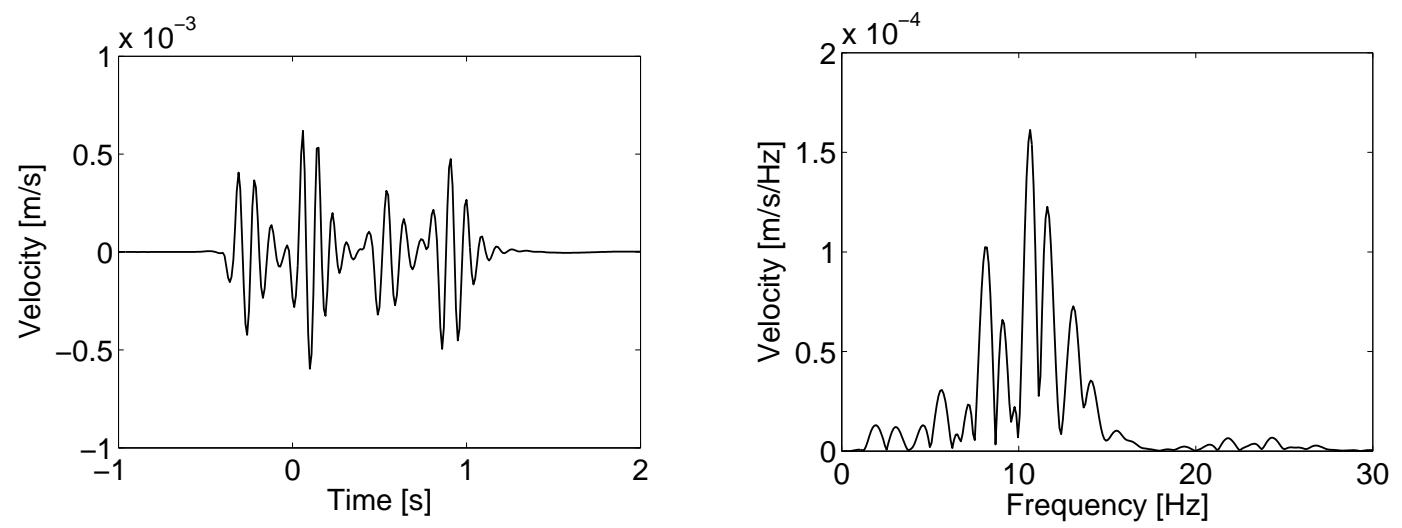

2.
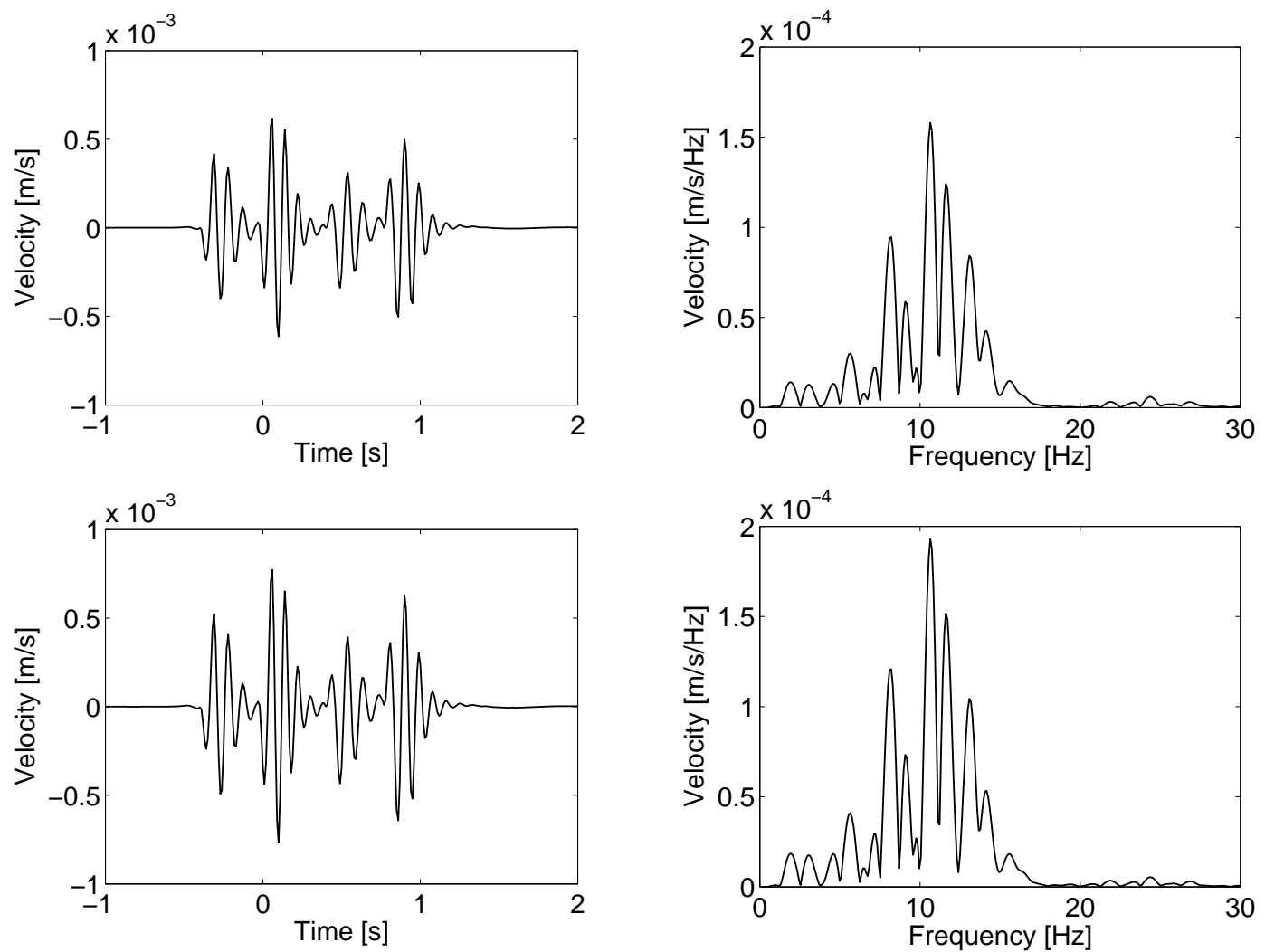

a. $v_{b x}(t)$.

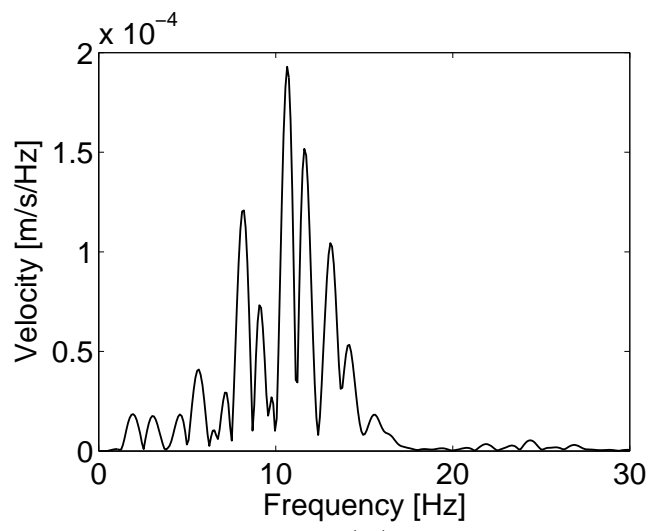

b. $\hat{v}_{b x}(\omega)$.

Figure 17: (a) Time history and (b) frequency content of the horizontal velocity in the point $\mathrm{M}$ on the edge between the front wall and the slab on the second floor for the structure with (1) a slab, (2) a strip and (3) a box foundation. 

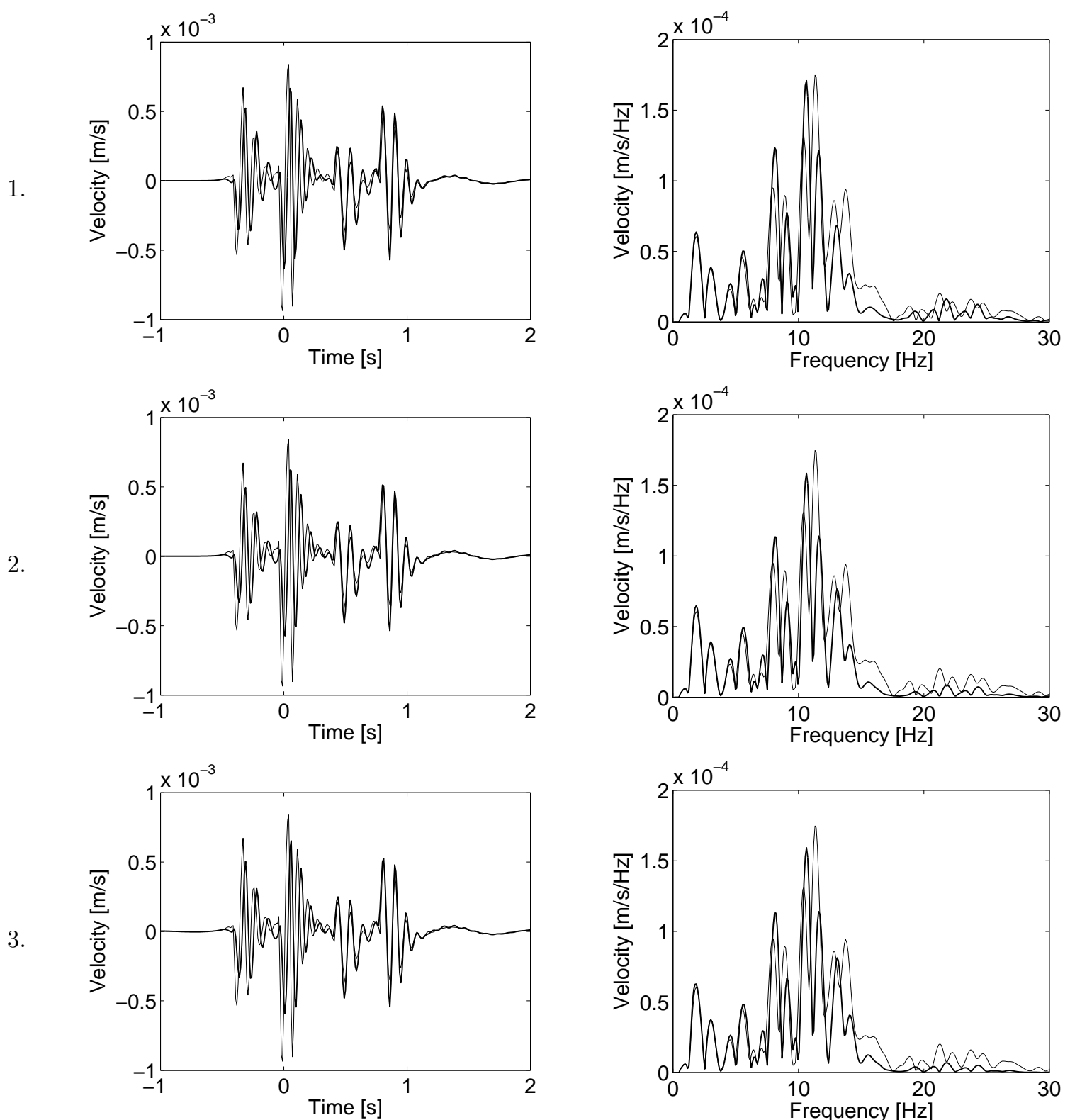

a. $v_{b z}(t)$.

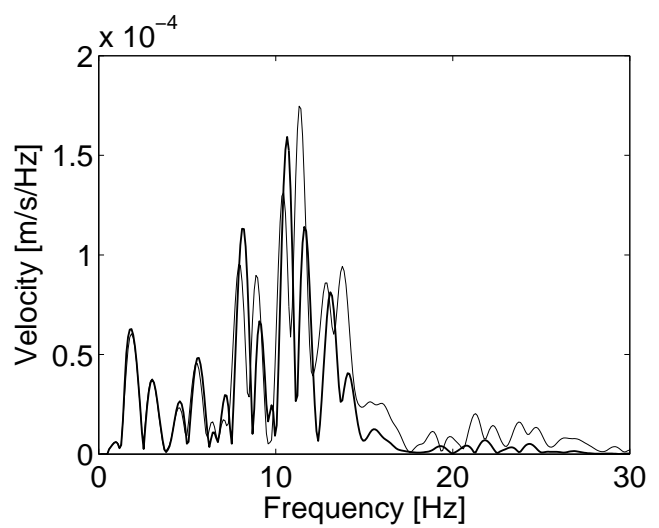

b. $\hat{v}_{b z}(\omega)$.

Figure 18: (a) Time history and (b) frequency content of the vertical velocity in the point I on the edge between the front wall and the foundation for the structure with (1) a slab, (2) a strip and (3) a box foundation. The incident wave field is indicated with a thin line. 
1.
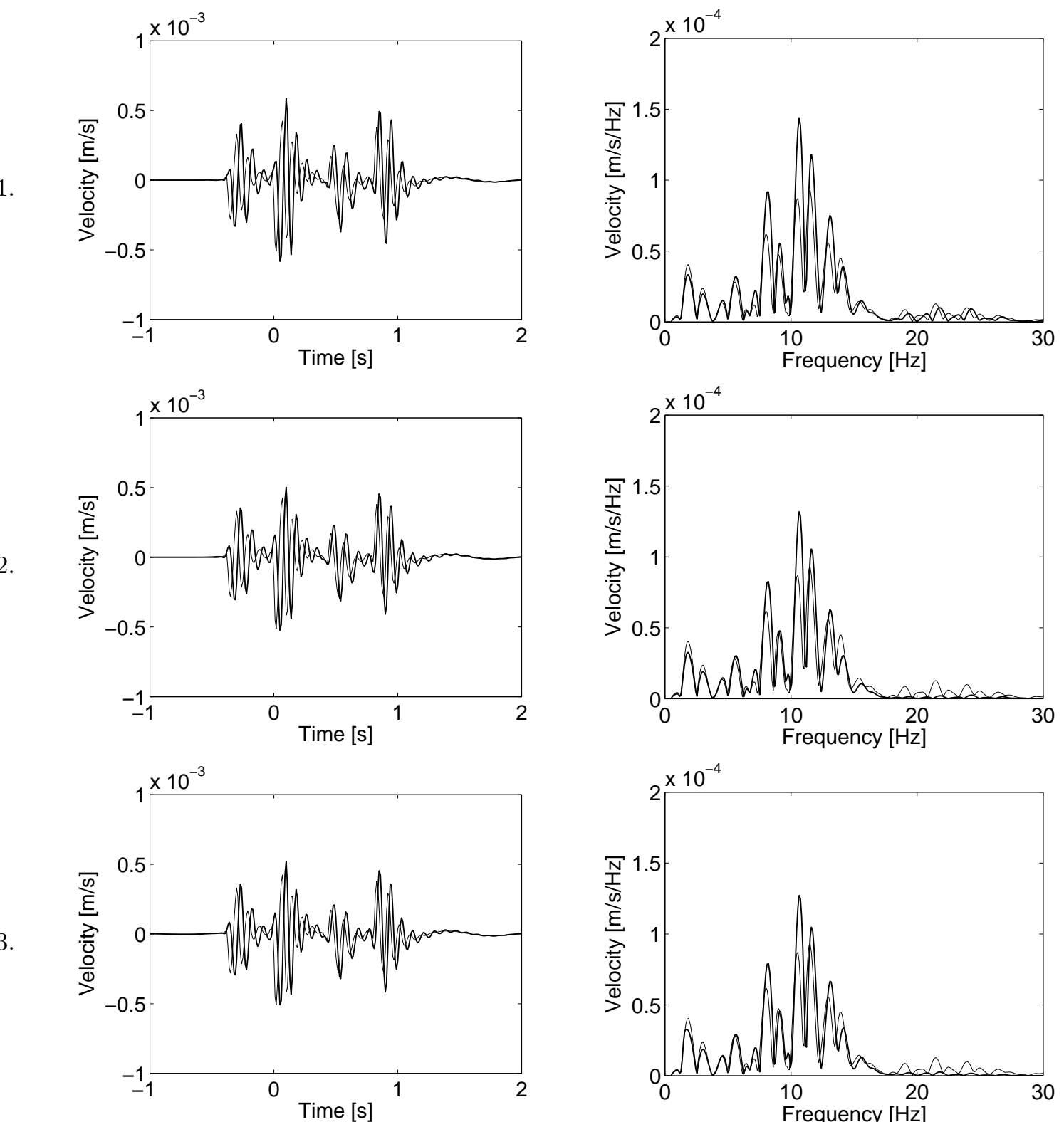

a. $v_{b z}(t)$.

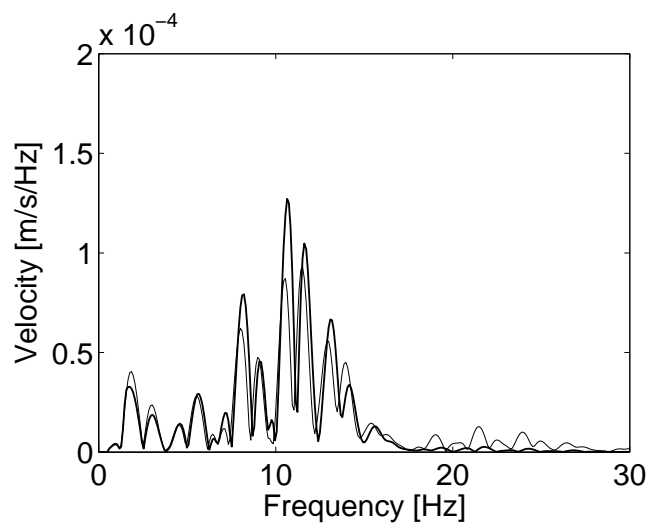

b. $\hat{v}_{b z}(\omega)$.

Figure 19: (a) Time history and (b) frequency content of the vertical velocity in the point $\mathrm{K}$ on the edge between the rear wall and the foundation for the structure with (1) a slab, (2) a strip and (3) a box foundation. The incident wave field is indicated with a thin line. 


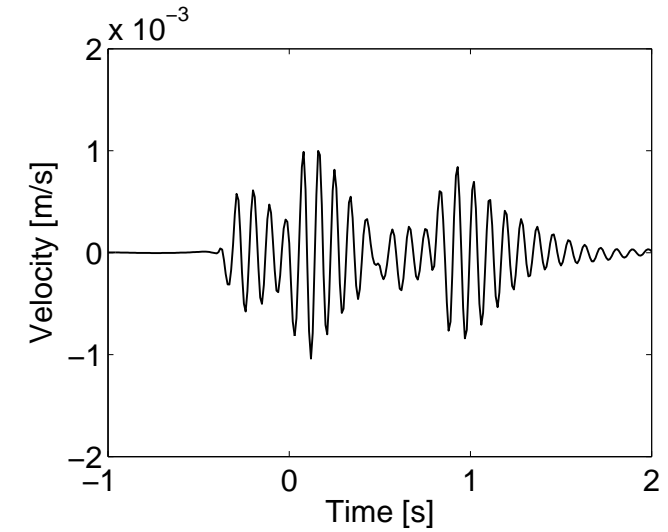

a. $v_{b z}(t)$.

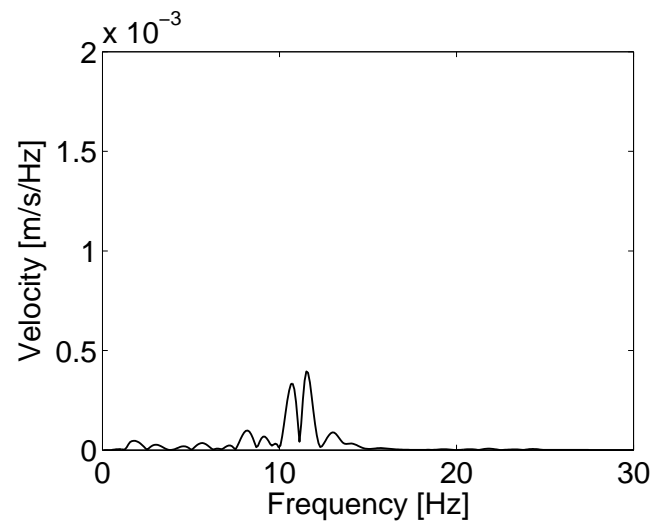

b. $\hat{v}_{b z}(\omega)$.

Figure 20: (a) Time history and (b) frequency content of the vertical velocity in point $\mathrm{L}$ in the centre of the slab on the first floor for the structure with a box foundation. 


\section{Conclusions}

A study of the dynamic response of buildings due to traffic induced vibrations has been performed, accounting for the effect of dynamic soil-structure interaction. The response of a two-storey single family dwelling due to traffic induced vibrations has been considered for three different foundation types. An investigation of the modes of the structure and the comparison of the response in different points in the structure enables to formulate the following conclusions:

1. In general, two situations may occur. (1) For a building resting on a soft soil, no wall deformation occurs. The global structural response is dominated by rigid body kinematics. (2) If the soil is stiff with respect to the structure, the walls deform in a quasi-static way, following the ground motion. Additionally, the presence of a (stiff) foundation prevents wall deformations. This has important consequences for vibration induced damage to buildings: wall cracking, caused by excessive deformations, is more likely to occur in the case of a soft structure on a stiff soil.

2. When no dynamic soil-structure interaction is accounted for, the assumption that the motion of the foundation is equal to the incident wave field results in unrealistic wall deformations when the structure is relatively rigid with respect to the soil. Allowing only for a rigid body motion of the foundation as a spatial average of the incident wave field, results in a better approximation of the wall displacements.

3. The response of the floors is dominated by local bending modes. Dynamic soil-structure interaction results in an increased attenuation of the floor response, as the energy in the structure can be dissipated through geometric damping in the soil.

\section{Acknowledgements}

The results presented in this paper have been obtained within the frame of the STWW-project IWT000152 'Traffic induced vibrations in buildings'. The financial support of the Ministry of the Flemish Community is gratefully acknowledged.

\section{References}

[1] L. Andersen and S.R.K. Nielsen. Boundary element analysis of the steady-state response of an elastic half-space to a moving force on its surface. Engineering Analysis with Boundary Elements, 27:23-38, 2003.

[2] H. Antes and C.C. Spyrakos. Soil-structure interaction. In D.E. Beskos and S.A. Anagnostopoulos, editors, Computer analysis and design of earthquake resistant structures: a handbook, volume 3 of Advances in Earthquake Engineering, pages 271-332, 1997.

[3] D. Aubry and D. Clouteau. A subdomain approach to dynamic soil-structure interaction. In V. Davidovici and R.W. Clough, editors, Recent advances in Earthquake Engineering and Structural Dynamics, pages 251-272. Ouest Editions/AFPS, Nantes, 1992.

[4] D.E. Beskos, T. Krauthammer, and I. Vardoulakis, editors. Dynamic soil-structure interaction. A.A. Balkema, 1984.

[5] M. Bonnet. Boundary integral equation methods for solids and fluids. John Wiley and Sons, Chichester, United Kingdom, 1995.

[6] D. Clouteau. Propagation d'ondes dans des milieux hétérogènes. Application à la tenue des ouvrages sous séismes. $\mathrm{PhD}$ thesis, Laboratoire de Mécanique des Sols, Structures et Matériaux, Ecole Centrale de Paris, 1990.

[7] D. Clouteau. MISS Revision 6.2, Manuel Scientifique. Laboratoire de Mécanique des Sols, Structures et Matériaux, Ecole Centrale de Paris, 1999.

[8] D. Clouteau. MISS Revision 6.2, Manuel Utilisateur. Laboratoire de Mécanique des Sols, Structures et Matériaux, Ecole Centrale de Paris, 1999.

[9] D. Clouteau, G. Degrande, and G. Lombaert. Numerical modelling of traffic induced vibrations. Meccanica, 36(4):401-420, 2001.

[10] R.J. Craig and M. Bampton. Coupling of substructures for dynamic analyses. AIAA Journal, 6(7):1313-1319, 1968.

[11] J. Domínguez. Boundary elements in dynamics. Computational Mechanics Publications and Elsevier Applied Science, Southampton, United Kingdom, 1993.

[12] T. Ekevid and N.-E. Wiberg. Wave propagation related to high-speed train. A scaled boundary FE-approach for unbounded domains. Computer Methods in Applied Mechanics and Engineering, 191:3947-3964, 2002.

[13] S. François, G. Lombaert, and G. Degrande. Local and global shape functions in a boundary element method for the calculation of traffic induced vibrations. Soil Dynamics and Earthquake Engineering, 25(11):839-856, 2005. 
[14] S. François, L. Pyl, H.R. Masoumi, and G. Degrande. Parametric study on determining factors for traffic induced vibrations in buildings. In P. Sas and M. De Munck, editors, ISMA2004 International Conference on Noise and Vibration Engineering, pages 2819-2834, Leuven, Belgium, September 2004.

[15] H. Grundmann, M. Lieb, and E. Trommer. The response of a layered half-space to traffic loads moving along its surface. Archive of Applied Mechanics, 69:55-67, 1999.

[16] G. Lombaert. Development and experimental validation of a numerical model for the free field vibrations induced by road traffic. PhD thesis, Department of Civil Engineering, KU Leuven, 2001.

[17] G. Lombaert and G. Degrande. Experimental validation of a numerical prediction model for free field traffic induced vibrations by in situ experiments. Soil Dynamics and Earthquake Engineering, 21(6):485-497, 2001.

[18] G. Lombaert and G. Degrande. The experimental validation of a numerical model for the prediction of the vibrations in the free field produced by road traffic. Journal of Sound and Vibration, 262:309-331, 2003.

[19] G. Lombaert, G. Degrande, and D. Clouteau. Numerical modelling of free field traffic induced vibrations. Soil Dynamics and Earthquake Engineering, 19(7):473-488, 2000.

[20] J.E. Luco. Linear soil-structure interaction : a review. Applied Mechanics Division, ASME, 53:41-57, 1982.

[21] J.E. Luco and H.L. Wong. Seismic response of foundations embedded in a layered half-space. Earthquake Engineering and Structural Dynamics, 15:233-247, 1987.

[22] A.V. Metrikine and K. Popp. Instability of vibrations of an oscillator moving along a beam on an elastic half-space. European Journal of Mechanics, A/Solids, 18(2):331-349, 1999.

[23] A.V. Metrikine, S.N. Verichev, and J. Blauwendraad. Stability of a two-mass oscillator moving on a beam supported by a visco-elastic half-space. International Journal of Solids and Structures, 42:1187-1207, 2005.

[24] N.N. Prognosemodel trillingshinder. Technical Report 95-2, Civieltechnisch Centrum Uitvoering Research en Regelgeving, February 1995.

[25] L. Pyl. Development and experimental validation of a numerical model for traffic induced vibrations in buildings. PhD thesis, Department of Civil Engineering, KU Leuven, 2004.

[26] L. Pyl, D. Clouteau, and G. Degrande. A weakly singular boundary integral equation in elastodynamics for heterogeneous domains mitigating fictitious eigenfrequencies. Engineering Analysis with Boundary Elements, 28(12):1493-1513, 2004.

[27] L. Pyl, G. Degrande, and D. Clouteau. Validation of a source-receiver model for road traffic induced vibrations in buildings. II: Receiver model. ASCE Journal of Engineering Mechanics, 130(12):1394-1406, 2004.

[28] L. Pyl, G. Degrande, G. Lombaert, and W. Haegeman. Validation of a source-receiver model for road traffic induced vibrations in buildings. I: Source model. ASCE Journal of Engineering Mechanics, 130(12):1377-1393, 2004.

[29] X. Sheng, C.J.C. Jones, and D.J. Thompson. A theoretical model for ground vibration from trains generated by vertical track irregularities. Journal of Sound and Vibration, 272(3-5):937-965, 2004.

[30] X. Sheng, C.J.C. Jones, and D.J. Thompson. A theoretical study of the influence of the track on train-induced ground vibration. Journal of Sound and Vibration, 272(3-5):909-936, 2004.

[31] X. Sheng, C.J.C. Jones, and D.J. Thompson. Responses of infinite periodic structures to moving or stationary harmonic loads. Journal of Sound and Vibration, 282:125-149, 2005.

[32] H. Takemiya. Simulation of track-ground vibrations due to a high-speed train: the case of X-2000 at Ledsgard. Journal of Sound and Vibration, 261:503-526, 2003.

[33] D.D. Theodorakopoulos and D.E. Beskos. Application of Biot's poroelasticity to some soil dynamics problems in civil engineering. Soil Dynamics and Earthquake Engineering, 26:666-679, 2006.

[34] A.S. Veletsos and J.W. Meek. Dynamic behaviour of building-foundation systems. Earthquake Engineering and Structural Dynamics, 3:121-138, 1974.

[35] G.R. Watts. Traffic-induced ground-borne vibrations in dwellings. Research report 102, Transport and Road Research Laboratory, 1987.

[36] J. P. Wolf and C. M. Song. Some cornerstones of dynamic soil-structure interaction. Engineering Structures, 24:13-28, 2002 .

[37] J.P. Wolf. Dynamic soil-structure interaction. Prentice-Hall, Englewood Cliffs, New Jersey, 1985. 